\title{
Budget outturns of Croatian municipalities, cities and counties for $2015^{1}$
}

\author{
KATARINA OTT, MiHAELA BRONIĆ, BRANKO STANIĆ
}

This article aims to provide a simple presentation of basic data on the budget outturns of Croatian local government units in 20I5. ${ }^{2}$ It includes synthesized tables based on the Ministry of Finance's database, showing revenues and expenditures ${ }^{3}$, as well as surpluses or deficits (in per capita and total terms). Also presented are data on the populations ${ }^{4}$ and levels of budget transparency in all the local government units ${ }^{5}$. This analysis and the tables additionally provided in the Excel format, allow all interested parties to get acquainted with the financial condition of their respective local government units in 2015 and make relevant comparisons with the situation in $2014^{6}$.

While the Ministry of Finance does publish budget outturns for all local government units , they are in the form of oversized Excel tables, with the cities and municipalities arranged by county. Given the large number of local government units (whose complete budgets are presented), the navigation through these tables and comparison across the local units is often difficult. This article gives the reader a snapshot of the basic financial condition of the local government units in 2015, while more detailed information can be found on the Ministry of Finance's and local government units' websites. Nevertheless, some notes may be necessary for a proper understanding of the presented data.

First, the amounts for counties, cities and municipalities should not be compared, because each of these types of local government units has the authority to collect different types of revenues and provide different

\footnotetext{
${ }^{1}$ This article was produced within the project Understanding, monitoring and analysing local government budget transparency: A case study of Croatia and Slovenia - Open local budget index (OLBI), IP-20I4-09-3008, funded by the Croatian Science Foundation.

${ }^{2}$ For the purposes of this article, the term „local government units” refers to all counties, cities and municipalities, and the term „local budgets” includes the budgets of all the counties, cities and municipalities.

${ }^{3}$ Receipts from financial assets and borrowing, and outlays on financial assets and loan repayment are not included. This analysis only covers revenues and expenditures, excluding receipts and outlays. The revenues and expenditures relate to business operations and the sale and acquisition of non-financial assets (e.g. land, buildings, concessions, vehicles, etc.), whereas receipts and outlays relate to financial assets, borrowing, lending and loan repayment. For more details, see A Citizen's Guide to the Budget.

${ }_{4}^{4}$ According to the CBS (2016).

5 The results of the analysis are available in Ott et al. (20I6) and at: http:/ /www.ijf.hr/transparency .

${ }^{6}$ The results for 2014 are available in Ott and Bronić (2OI5).

7 The Ministry of Finance's website (2016) offers the data for 1998-2015.
} 
kinds of public goods and services. Hence, different average per capita expenditures in counties (HRK I,O77), municipalities (HRK 3,296) and cities (HRK 3,876), indicate differences in authority across these local units . $^{2}$ Any person can add up the per capita revenues or expenditures in their respective municipality/city and county, to establish the total per capita revenues or expenditures at the level of that municipality or city. The City of Zagreb is an exception, as it is both a city and a county. Its citizens should therefore only consider the data for Zagreb.

Secondly, it is noteworthy that the budget does not always reflect the actual financial condition of a local government unit, because local government units sometimes take out loans through legal persons in their majority or co-ownership, or institutions founded by them ${ }^{9}$, rather than through their own budgets. Consequently, to establish the actual financial condition of a local government unit, one should have access not only to the local budget data, but also data on the revenues/expenditures, assets, capital and liabilities of the legal persons in their majority or co-ownership (e.g. utility companies), as well as institutions founded by them (e.g. sports facility management institutions). Regrettably, the data on such companies and institutions are not publicly available in one place.

Thirdly, sharp differences between revenues and expenditures in a given year may not be an indicator of bad planning or poor local budget outturns, but rather the consequence of, e.g., large capital asset sales (e.g. the sales of land or buildings), large-scale capital investments (e.g. in local infrastructure), funded from loans or EU aid, or transfers of revenue/receipt surpluses/deficits from previous years ${ }^{\mathrm{IO}}$. Part of these data for each of the local government units can be found on the Ministry of Finance's website, i.e. in an archive of budget outturns for all local units. However, for a full picture of a local government unit's financial condition, the Ministry should, in addition to the revenue and expenditure outturns data, also publish figures from the balance sheets of all the local government units on an annual basis and in one place, i.e. the data on their assets, liabilities and capital. ${ }^{\text {I }}$ This is because the real and accurate financial condition of a local unit can hardly be established only on the basis of budget data (i.e. revenues/receipts and expenditures/outlays) and without the data on assets, liabilities and capital.

While the aim of this article is not to analyse the financial condition of local government units in 2015, but rather to provide a synthesis of data from the Ministry of Finance's database, note should be taken of some interesting facts that immediately catch the eye:

- All fifty municipalities with the highest per capita revenues, ranging from HRK 2I,756 (Janjina) to HRK 5,939 (Mljet) are located in the Adriatic Croatia, 4I of them along the seacoast.

- Only five out of a hundred municipalities with the lowest per capita revenues are in the Adriatic Croatia, yet none of them being situated along the seacoast; about 30\% of them are in the BrodPosavina and Vukovar-Srijem counties and none in the Istria, Lika-Senj, Primorje-Gorski Kotar and Šibenik-Knin counties.

- All twenty cities with the highest per capita revenues, ranging from HRK II,2II (Mali Lošinj) to HRK 6,33I (Biograd na Moru), except two (Vrlika and Zagreb), are coastal cities; there are no cities along the seacoast with per capita revenues below HRK 2,00o.

- There are huge differences among municipalities in terms of total revenues, so that the total revenues of 30 poorest municipalities together equal the revenue of only one of the three richest municipalities, i.e. Konavle, Vir and Medulin (about HRK 6om each).

\footnotetext{
${ }^{8}$ Given its double status as a city and a county, performing the functions of both of these types of units, Zagreb is not included in either the county or city average. Its per capita expenditure (HRK 7,719) already deviates markedly from both the city and county averages.

${ }^{9}$ For more information on the borrowing of companies (co-)owned by local government units, see Bajo and Primorac (2OI4).

${ }^{\text {Io }}$ For more information on the transfer of revenue and receipt surpluses/deficits from previous years see link.

${ }^{\text {II }}$ Part of these data may already, or will soon be available on the local government units' websites, because, as of 20I5, all local government units, as well as budget and extra-budgetary users are required, pursuant to Article I2 of the Budget Act, to publish their annual financial statements on the official websites no later than eight days from their submission to the Financial Agency.
} 
- Total revenues of the poorest municipalities are below HRK Im, and per capita revenues below HRK I,OOO (as compared with the highest per capita revenue of HRK 2I,756 reported by the municipality of Janjina, or with the per capita revenue average of HRK 3,328 for municipalities).

- Total revenues of about $30 \%$ of cities with the lowest total revenues (43 of them) together equal the total revenues of Rijeka or Split (about HRK 68om each).

- So, there are cities with total revenues lower than HRK Iom (Kutjevo, Opuzen and Klanjec), but also municipalities with total revenues as high as about HRK 6om.

- As many as 42 cities or municipalities have higher per capita revenues than the City of Zagreb (HRK 7,856), e.g. the municipalities of Janjina (HRK 21,756), Vir (HRK 19,987), Baška (HRK 16,938), Mošćenička Draga (HRK I6,696), Sutivan (HRK I6,493) and Grožnjan (HRK 15,949).

- Fifty-two municipalities have a population of over 5,000, six of them having more than I0,000 inhabitants, while 60 cities have a population below IO,OOO, I8 of them having less than 5,000 inhabitants.

- Some municipalities and cities stand out for a substantial excess of total expenditures over revenues, e.g. the municipalities of Smokvica (I86\%), Pokupsko (167\%), Jalžabet (137\%), KaštelirLabinci (II7\%) and Pojezerje (II2\%), and the cities of Supetar (37\%) and Ozalj (33\%).

- Standing out for major surpluses of total revenues over expenditures are the following municipalities: Sveti Petar u Šumi (IOO\%), Breznica (83\%), Gornji Kneginec (80\%), Legrad (79\%), Luka (70\%), Vir (69\%), Jakšić (67\%) and Primošten (59\%), and the city of Zlatar (57\%).

In keeping with the motto „If you collect and process data, publish them; if the data are not published, demand them to be published and once they are published, use them!", tables are also provided in the Excel format. Those interested can download them to their own computers for further use and rearrangement.

It can be assumed that this synthesis (which is also an analysis in itself) of the extensive Ministry of Finance's database will prompt readers to visit the database, in order to get detailed information on their local government units' budgets and to participate in analysing and deciding on the collection and spending of the local budget funds. It is also hoped that it will serve as a basis for an analysis that should precede decisions on a potential territorial and fiscal reorganisation of the country.

In order to provide a real insight into the financial condition of local government units, the Ministry of Finance should start publishing, (either individually or in collaboration with the Ministry of Administration), in one place and in a machine-readable format: I) data on revenues and expenditures, as well as assets, liabilities and capital of legal persons in majority ownership/co-ownership of local units and institutions founded by them; and 2) balance sheets of all local government units, including data on their respective assets, liabilities and capital. 
Table I.

Budget outturns of municipalities, 2015

Per capita (in HRK)

Total (in million HRK)

\begin{tabular}{|c|c|c|c|c|c|c|c|c|c|}
\hline & Municipality & $\begin{array}{l}\text { Reve- } \\
\text { nues }\end{array}$ & $\begin{array}{l}\text { Expen- } \\
\text { ditures }\end{array}$ & $\begin{array}{l}\text { Surplus } \\
\text { /deficit }\end{array}$ & $\begin{array}{c}\text { Popula- } \\
\text { tion }\end{array}$ & $\begin{array}{c}\text { Reve- } \\
\text { nues }\end{array}$ & $\begin{array}{l}\text { Expen- } \\
\text { ditures }\end{array}$ & $\begin{array}{l}\text { Surplus } \\
\text { /deficit }\end{array}$ & $\begin{array}{l}\text { Trans- } \\
\text { paren- } \\
\text { cy }\end{array}$ \\
\hline 1 & Janjina & 21,756 & 22,372 & -616 & 551 & 12.0 & 12.3 & -0.3 & 1 \\
\hline 2 & Vir & 19,987 & 11,815 & 8,172 & 3,000 & 60.0 & 35.4 & 24.5 & 3 \\
\hline 3 & Baška & 16,938 & 15,044 & 1,894 & 1,674 & 28.4 & 25.2 & 3.2 & 4 \\
\hline 4 & Mošćenička Draga & 16,696 & 10,643 & 6,052 & 1,535 & 25.6 & 16.3 & 9.3 & 4 \\
\hline 5 & Sutivan & 16,493 & 13,548 & 2,945 & 822 & 13.6 & 11.1 & 2.4 & 0 \\
\hline 6 & Grožnjan & 15,949 & 10,670 & 5,279 & 736 & 11.7 & 7.9 & 3.9 & 4 \\
\hline 7 & Funtana & 12,987 & 12,643 & 344 & 907 & 11.8 & 11.5 & 0.3 & 3 \\
\hline 8 & Kostrena & 11,508 & 11,469 & 39 & 4,180 & 48.1 & 47.9 & 0.2 & 5 \\
\hline 9 & Kolan & 11,488 & 8,574 & 2,914 & 791 & 9.1 & 6.8 & 2.3 & 0 \\
\hline 10 & Tisno & 11,406 & 10,658 & 748 & 3,094 & 35.3 & 33.0 & 2.3 & 2 \\
\hline 11 & Bale & 11,327 & 10,462 & 865 & 1,127 & 12.8 & 11.8 & 1.0 & 2 \\
\hline 12 & $\begin{array}{l}\text { Malinska - } \\
\text { Dubašnica }\end{array}$ & 10,726 & 10,080 & 645 & 3,134 & 33.6 & 31.6 & 2.0 & 0 \\
\hline 13 & Vrsar & 10,598 & 8,681 & 1,917 & 2,162 & 22.9 & 18.8 & 4.1 & 1 \\
\hline 14 & Omišalj & 10,189 & 10,371 & -182 & 2,983 & 30.4 & 30.9 & -0.5 & 3 \\
\hline 15 & Lastovo & 9,671 & 10,491 & -820 & 792 & 7.7 & 8.3 & -0.6 & 1 \\
\hline 16 & Lopar & 9,423 & 8,537 & 886 & 1,263 & 11.9 & 10.8 & 1.1 & 1 \\
\hline 17 & Šolta & 9,250 & 7,713 & 1,537 & 1,700 & 15.7 & 13.1 & 2.6 & 1 \\
\hline 18 & Medulin & 9,235 & 9,385 & -150 & 6,481 & 59.8 & 60.8 & -1.0 & 3 \\
\hline 19 & Primošten & 9,197 & 5,766 & 3,431 & 2,828 & 26.0 & 16.3 & 9.7 & 2 \\
\hline 20 & Dugopolje & 9,089 & 7,049 & 2,040 & 3,469 & 31.5 & 24.5 & 7.1 & 1 \\
\hline 21 & Bol & 9,076 & 11,570 & $-2,494$ & 1,630 & 14.8 & 18.9 & -4.1 & 0 \\
\hline 22 & Lupoglav & 8,969 & 7,062 & 1,907 & 924 & 8.3 & 6.5 & 1.8 & 3 \\
\hline 23 & Tar-Vabriga & 8,803 & 9,634 & -832 & 1,990 & 17.5 & 19.2 & -1.7 & 4 \\
\hline 24 & Brtonigla & 8,751 & 8,552 & 199 & 1,626 & 14.2 & 13.9 & 0.3 & 3 \\
\hline 25 & Punat & 8,626 & 9,226 & -600 & 1,973 & 17.0 & 18.2 & -1.2 & 4 \\
\hline 26 & Jasenice & 8,539 & 7,710 & 828 & 1,398 & 11.9 & 10.8 & 1.2 & 0 \\
\hline 27 & Baška Voda & 8,435 & 6,021 & 2,414 & 2,775 & 23.4 & 16.7 & 6.7 & 1 \\
\hline 28 & Pašman & 8,430 & 5,378 & 3,052 & 2,082 & 17.6 & 11.2 & 6.4 & 0 \\
\hline 29 & Povljana & 8,208 & 8,716 & -508 & 759 & 6.2 & 6.6 & -0.4 & 0 \\
\hline 30 & Lovinac & 8,120 & 10,211 & $-2,091$ & 1,007 & 8.2 & 10.3 & -2.1 & 3 \\
\hline 31 & Civljane & 8,034 & 7,474 & 560 & 239 & 1.9 & 1.8 & 0.1 & 3 \\
\hline 32 & Rogoznica & 7,901 & 6,594 & 1,308 & 2,345 & 18.5 & 15.5 & 3.1 & 2 \\
\hline 33 & Fažana & 7,620 & 7,953 & -332 & 3,635 & 27.7 & 28.9 & -1.2 & 4 \\
\hline 34 & Fužine & 7,602 & 7,671 & -69 & 1,592 & 12.1 & 12.2 & -0.1 & 0 \\
\hline 35 & Lećevica & 7,381 & 6,308 & 1,073 & 583 & 4.3 & 3.7 & 0.6 & 2 \\
\hline 36 & Kršan & 7,379 & 7,156 & 223 & 2,951 & 21.8 & 21.1 & 0.7 & 4 \\
\hline 37 & Karlobag & 7,129 & 11,218 & $-4,090$ & 917 & 6.5 & 10.3 & -3.8 & 0 \\
\hline 38 & Konavle & 7,065 & 5,102 & 1,963 & 8,577 & 60.6 & 43.8 & 16.8 & 5 \\
\hline 39 & Nerežišća & 6,995 & 6,106 & 888 & 862 & 6.0 & 5.3 & 0.8 & 0 \\
\hline 40 & Župa Dubrovačka & 6,994 & 7,065 & -71 & 8,331 & 58.3 & 58.9 & -0.6 & 1 \\
\hline 41 & Oprtalj & 6,882 & 4,349 & 2,533 & 850 & 5.8 & 3.7 & 2.2 & 2 \\
\hline 42 & Zadvarje & 6,862 & 8,048 & $-1,185$ & 289 & 2.0 & 2.3 & -0.3 & 1 \\
\hline 43 & Dobrinj & 6,792 & 6,533 & 259 & 2,078 & 14.1 & 13.6 & 0.5 & 3 \\
\hline 44 & Vrbnik & 6,785 & 8,205 & $-1,419$ & 1,260 & 8.5 & 10.3 & -1.8 & 4 \\
\hline 45 & Tučepi & 6,702 & 5,846 & 856 & 1,931 & 12.9 & 11.3 & 1.7 & 0 \\
\hline 46 & Lovran & 6,691 & 7,328 & -636 & 4,101 & 27.4 & 30.1 & -2.6 & 5 \\
\hline 47 & Kijevo & 6,327 & 5,757 & 570 & 417 & 2.6 & 2.4 & 0.2 & 2 \\
\hline 48 & Ston & 6,151 & 7,170 & $-1,019$ & 2,407 & 14.8 & 17.3 & -2.5 & 3 \\
\hline 49 & Starigrad & 6,023 & 4,946 & 1,077 & 1,876 & 11.3 & 9.3 & 2.0 & 4 \\
\hline 50 & Mljet & 5,939 & 5,926 & 13 & 1,088 & 6.5 & 6.4 & 0.0 & 2 \\
\hline 51 & Milna & 5,923 & 4,491 & 1,433 & 1,034 & 6.1 & 4.6 & 1.5 & 1 \\
\hline 52 & Podgora & 5,902 & 5,464 & 439 & 2,518 & 14.9 & 13.8 & 1.1 & 2 \\
\hline 53 & Motovun & 5,838 & 9,507 & $-3,669$ & 1,004 & 5.9 & 9.5 & -3.7 & 3 \\
\hline
\end{tabular}

4 NEWSLETTER Io8 | K. OTT, M. BRONIĆ, B. STANIĆ | Budget outturns of Croatian municipalities... | Institute of Public Finance 
Per capita (in HRK)

Total (in million HRK)

\begin{tabular}{|c|c|c|c|c|c|c|c|c|c|}
\hline & Municipality & $\begin{array}{l}\text { Reve- } \\
\text { nues }\end{array}$ & $\begin{array}{l}\text { Expen- } \\
\text { ditures }\end{array}$ & $\begin{array}{l}\text { Surplus } \\
\text { /deficit }\end{array}$ & $\begin{array}{l}\text { Popula- } \\
\text { tion }\end{array}$ & $\begin{array}{l}\text { Reve- } \\
\text { nues }\end{array}$ & $\begin{array}{l}\text { Expen- } \\
\text { ditures }\end{array}$ & $\begin{array}{l}\text { Surplus } \\
\text { /deficit }\end{array}$ & $\begin{array}{l}\text { Trans- } \\
\text { paren- } \\
\text { cy }\end{array}$ \\
\hline 54 & Lanišće & 5,833 & 5,913 & -80 & 329 & 1.9 & 1.9 & 0.0 & 2 \\
\hline 55 & Tribunj & 5,648 & 5,608 & 40 & 1,536 & 8.7 & 8.6 & 0.1 & 3 \\
\hline 56 & Murter & 5,599 & 4,685 & 913 & 2,044 & 11.4 & 9.6 & 1.9 & 1 \\
\hline 57 & Brela & 5,581 & 6,354 & -773 & 1,703 & 9.5 & 10.8 & -1.3 & 2 \\
\hline 58 & Luka & 5,512 & 3,235 & 2,277 & 1,351 & 7.4 & 4.4 & 3.1 & 3 \\
\hline 59 & Okrug & 5,378 & 5,365 & 13 & 3,349 & 18.0 & 18.0 & 0.0 & 4 \\
\hline 60 & Sućuraj & 5,347 & 6,795 & $-1,448$ & 463 & 2.5 & 3.1 & -0.7 & 0 \\
\hline 61 & Kukljica & 5,257 & 4,998 & 259 & 714 & 3.8 & 3.6 & 0.2 & 1 \\
\hline 62 & Pakoštane & 5,256 & 4,107 & 1,149 & 4,123 & 21.7 & 16.9 & 4.7 & 1 \\
\hline 63 & Vrsi & 5,219 & 4,857 & 363 & 2,053 & 10.7 & 10.0 & 0.7 & 0 \\
\hline 64 & Kalinovac & 5,219 & 6,160 & -941 & 1,597 & 8.3 & 9.8 & -1.5 & 4 \\
\hline 65 & Tkon & 5,142 & 5,978 & -836 & 763 & 3.9 & 4.6 & -0.6 & 1 \\
\hline 66 & Saborsko & 5,068 & 5,831 & -763 & 632 & 3.2 & 3.7 & -0.5 & 2 \\
\hline 67 & $\begin{array}{l}\text { Dubrovačko } \\
\text { Primorje }\end{array}$ & 5,018 & 6,193 & $-1,175$ & 2,170 & 10.9 & 13.4 & -2.5 & 1 \\
\hline 68 & Postira & 4,948 & 4,662 & 285 & 1,559 & 7.7 & 7.3 & 0.4 & 2 \\
\hline 69 & Ribnik & 4,932 & 3,918 & 1,014 & 475 & 2.3 & 1.9 & 0.5 & 4 \\
\hline 70 & Pirovac & 4,872 & 5,574 & -702 & 1,930 & 9.4 & 10.8 & -1.4 & 4 \\
\hline 71 & Sveti Petar u Šumi & 4,865 & 2,429 & 2,437 & 1,065 & 5.2 & 2.6 & 2.6 & 1 \\
\hline 72 & Sveti Lovreč & 4,816 & 4,992 & -176 & 1,015 & 4.9 & 5.1 & -0.2 & 3 \\
\hline 73 & Primorski Dolac & 4,752 & 6,675 & $-1,922$ & 770 & 3.7 & 5.1 & -1.5 & 3 \\
\hline 74 & Plitvička Jezera & 4,672 & 7,799 & $-3,127$ & 4,373 & 20.4 & 34.1 & -13.7 & 1 \\
\hline 75 & Stupnik & 4,669 & 5,419 & -750 & 3,735 & 17.4 & 20.2 & -2.8 & 3 \\
\hline 76 & Kanfanar & 4,610 & 4,516 & 94 & 1,543 & 7.1 & 7.0 & 0.1 & 2 \\
\hline 77 & Promina & 4,579 & 4,890 & -311 & 1,136 & 5.2 & 5.6 & -0.4 & 3 \\
\hline 78 & Pisarovina & 4,559 & 5,125 & -566 & 3,689 & 16.8 & 18.9 & -2.1 & 4 \\
\hline 79 & Draž & 4,497 & 4,226 & 272 & 2,767 & 12.4 & 11.7 & 0.8 & 0 \\
\hline 80 & Vižinada & 4,427 & 7,406 & $-2,979$ & 1,158 & 5.1 & 8.6 & -3.4 & 2 \\
\hline 81 & Prgomet & 4,417 & 4,352 & 65 & 673 & 3.0 & 2.9 & 0.0 & 0 \\
\hline 82 & Mrkopalj & 4,394 & 4,600 & -206 & 1,214 & 5.3 & 5.6 & -0.3 & 2 \\
\hline 83 & Polača & 4,374 & 4,015 & 360 & 1,468 & 6.4 & 5.9 & 0.5 & 2 \\
\hline 84 & Jagodnjak & 4,288 & 4,334 & -46 & 2,023 & 8.7 & 8.8 & -0.1 & 3 \\
\hline 85 & Raša & 4,270 & 4,436 & -166 & 3,183 & 13.6 & 14.1 & -0.5 & 4 \\
\hline 86 & Molve & 4,263 & 5,143 & -880 & 2,189 & 9.3 & 11.3 & -1.9 & 4 \\
\hline 87 & Ervenik & 4,226 & 4,574 & -349 & 1,105 & 4.7 & 5.1 & -0.4 & 2 \\
\hline 88 & Magadenovac & 4,217 & 4,656 & -439 & 1,936 & 8.2 & 9.0 & -0.8 & 2 \\
\hline 89 & Matulji & 4,206 & 4,330 & -124 & 11,246 & 47.3 & 48.7 & -1.4 & 4 \\
\hline 90 & Sveti Filip i Jakov & 4,198 & 4,308 & -111 & 4,606 & 19.3 & 19.8 & -0.5 & 3 \\
\hline 91 & Kali & 4,174 & 4,235 & -61 & 1,638 & 6.8 & 6.9 & -0.1 & 4 \\
\hline 92 & Jelsa & 4,131 & 4,337 & -206 & 3,582 & 14.8 & 15.5 & -0.7 & 1 \\
\hline 93 & Trpanj & 4,006 & 4,186 & -180 & 721 & 2.9 & 3.0 & -0.1 & 4 \\
\hline 94 & Privlaka (Zadar) & 4,005 & 3,773 & 232 & 2,253 & 9.0 & 8.5 & 0.5 & 4 \\
\hline 95 & Bistra & 4,001 & 3,911 & 90 & 6,632 & 26.5 & 25.9 & 0.6 & 3 \\
\hline 96 & Pićan & 3,903 & 5,000 & $-1,097$ & 1,827 & 7.1 & 9.1 & -2.0 & 3 \\
\hline 97 & Hrvace & 3,900 & 4,219 & -320 & 3,617 & 14.1 & 15.3 & -1.2 & 0 \\
\hline 98 & Marčana & 3,895 & 4,313 & -418 & 4,253 & 16.6 & 18.3 & -1.8 & 2 \\
\hline 99 & $\begin{array}{l}\text { Preko } \\
\end{array}$ & 3,818 & 5,881 & $-2,063$ & 3,805 & 14.5 & 22.4 & -7.8 & 2 \\
\hline 100 & Kamanje & 3,802 & 3,353 & 449 & 891 & 3.4 & 3.0 & 0.4 & 4 \\
\hline 101 & Veliki Grđevac & 3,798 & 3,978 & -181 & 2,849 & 10.8 & 11.3 & -0.5 & 2 \\
\hline 102 & Sali & 3,783 & 4,038 & -255 & 1,698 & 6.4 & 6.9 & -0.4 & 2 \\
\hline 103 & Vinodolska općina & 3,776 & 4,164 & -388 & 3,577 & 13.5 & 14.9 & -1.4 & 4 \\
\hline 104 & Kalnik & 3,772 & 4,909 & $-1,137$ & 1,351 & 5.1 & 6.6 & -1.5 & 2 \\
\hline 105 & Rakovica & 3,754 & 3,638 & 116 & 2,387 & 9.0 & 8.7 & 0.3 & 4 \\
\hline 106 & Ljubešćica & 3,748 & 3,742 & 6 & 1,858 & 7.0 & 7.0 & 0.0 & 3 \\
\hline 107 & Skrad & 3,736 & 3,772 & -35 & 1,062 & 4.0 & 4.0 & 0.0 & 2 \\
\hline 108 & Podstrana & 3,733 & 3,850 & -117 & 9,129 & 34.1 & 35.1 & -1.1 & 1 \\
\hline
\end{tabular}


Per capita (in HRK)

Total (in million HRK)

\begin{tabular}{|c|c|c|c|c|c|c|c|c|c|}
\hline & Municipality & $\begin{array}{l}\text { Reve- } \\
\text { nues }\end{array}$ & $\begin{array}{l}\text { Expen- } \\
\text { ditures }\end{array}$ & $\begin{array}{l}\text { Surplus } \\
\text { /deficit }\end{array}$ & $\begin{array}{l}\text { Popula- } \\
\text { tion }\end{array}$ & $\begin{array}{l}\text { Reve- } \\
\text { nues }\end{array}$ & $\begin{array}{l}\text { Expen- } \\
\text { ditures }\end{array}$ & $\begin{array}{l}\text { Surplus } \\
\text { /deficit }\end{array}$ & $\begin{array}{l}\text { Trans- } \\
\text { paren- } \\
\text { cy }\end{array}$ \\
\hline 109 & Orebić & 3,725 & 4,097 & -372 & 4,122 & 15.4 & 16.9 & -1.5 & 2 \\
\hline 110 & Petlovac & 3,705 & 2,515 & 1,189 & 2,405 & 8.9 & 6.0 & 2.9 & 2 \\
\hline 111 & Poličnik & 3,693 & 4,023 & -330 & 4,469 & 16.5 & 18.0 & -1.5 & 2 \\
\hline 112 & $\begin{array}{l}\text { Čavle } \\
\end{array}$ & 3,693 & 3,407 & 286 & 7,220 & 26.7 & 24.6 & 2.1 & 3 \\
\hline 113 & Gola & 3,682 & 3,414 & 268 & 2,431 & 9.0 & 8.3 & 0.7 & 3 \\
\hline 114 & Gunja & 3,667 & 3,453 & 214 & 3,732 & 13.7 & 12.9 & 0.8 & 2 \\
\hline 115 & Lokve & 3,664 & 4,048 & -384 & 1,049 & 3.8 & 4.2 & -0.4 & 0 \\
\hline 116 & Klana & 3,640 & 3,094 & 546 & 1,975 & 7.2 & 6.1 & 1.1 & 3 \\
\hline 117 & Zagvozd & 3,631 & 3,112 & 519 & 1,188 & 4.3 & 3.7 & 0.6 & 1 \\
\hline 118 & Majur & 3,630 & 3,346 & 285 & 1,185 & 4.3 & 4.0 & 0.3 & 2 \\
\hline 119 & Drenovci & 3,576 & 4,117 & -540 & 5,174 & 18.5 & 21.3 & -2.8 & 3 \\
\hline 120 & Ližnjan & 3,566 & 4,731 & $-1,165$ & 3,965 & 14.1 & 18.8 & -4.6 & 3 \\
\hline 121 & Gračišće & 3,544 & 2,655 & 889 & 1,419 & 5.0 & 3.8 & 1.3 & 4 \\
\hline 122 & Tinjan & 3,542 & 3,444 & 98 & 1,684 & 6.0 & 5.8 & 0.2 & 0 \\
\hline 123 & Zemunik Donji & 3,541 & 5,412 & $-1,871$ & 2,060 & 7.3 & 11.1 & -3.9 & 2 \\
\hline 124 & Marina & 3,503 & 3,435 & 68 & 4,595 & 16.1 & 15.8 & 0.3 & 1 \\
\hline 125 & Barban & 3,481 & 2,628 & 853 & 2,721 & 9.5 & 7.2 & 2.3 & 3 \\
\hline 126 & Lišane Ostrovičke & 3,440 & 4,225 & -785 & 698 & 2.4 & 2.9 & -0.5 & 0 \\
\hline 127 & Lumbarda & 3,421 & 3,955 & -534 & 1,213 & 4.1 & 4.8 & -0.6 & 3 \\
\hline 128 & Marija Gorica & 3,408 & 4,057 & -649 & 2,233 & 7.6 & 9.1 & -1.4 & 1 \\
\hline 129 & $\begin{array}{l}\text { Sveta Nedelja } \\
\text { (Istria) }\end{array}$ & 3,406 & 3,561 & -155 & 2,987 & 10.2 & 10.6 & -0.5 & 4 \\
\hline 130 & Kaštelir - Labinci & 3,400 & 7,392 & $-3,992$ & 1,463 & 5.0 & 10.8 & -5.8 & 0 \\
\hline 131 & Lovas & 3,394 & 3,275 & 119 & 1,214 & 4.1 & 4.0 & 0.1 & 2 \\
\hline 132 & Udbina & 3,366 & 2,994 & 372 & 1,874 & 6.3 & 5.6 & 0.7 & 4 \\
\hline 133 & Svetvinčenat & 3,365 & 3,091 & 274 & 2,202 & 7.4 & 6.8 & 0.6 & 1 \\
\hline 134 & Ravna Gora & 3,340 & 3,167 & 173 & 2,430 & 8.1 & 7.7 & 0.4 & 3 \\
\hline 135 & Nijemci & 3,298 & 3,571 & -273 & 4,705 & 15.5 & 16.8 & -1.3 & 3 \\
\hline 136 & Selca & 3,297 & 3,647 & -351 & 1,804 & 5.9 & 6.6 & -0.6 & 4 \\
\hline 137 & Gradac & 3,293 & 3,161 & 133 & 3,261 & 10.7 & 10.3 & 0.4 & 3 \\
\hline 138 & Stubičke Toplice & 3,289 & 3,290 & -1 & 2,805 & 9.2 & 9.2 & 0.0 & 4 \\
\hline 139 & Bibinje & 3,281 & 3,257 & 24 & 3,985 & 13.1 & 13.0 & 0.1 & 0 \\
\hline 140 & Viškovo & 3,254 & 3,523 & -269 & 14,445 & 47.0 & 50.9 & -3.9 & 4 \\
\hline 141 & Legrad & 3,254 & 1,817 & 1,437 & 2,241 & 7.3 & 4.1 & 3.2 & 4 \\
\hline 142 & Sirač & 3,231 & 4,111 & -881 & 2,218 & 7.2 & 9.1 & -2.0 & 3 \\
\hline 143 & Antunovac & 3,199 & 3,132 & 68 & 3,703 & 11.8 & 11.6 & 0.3 & 4 \\
\hline 144 & Kneževi Vinogradi & 3,187 & 3,239 & -51 & 4,614 & 14.7 & 14.9 & -0.2 & 2 \\
\hline 145 & Čeminac & 3,172 & 3,296 & -124 & 2,909 & 9.2 & 9.6 & -0.4 & 1 \\
\hline 146 & Tovarnik & 3,160 & 2,821 & 339 & 2,775 & 8.8 & 7.8 & 0.9 & 2 \\
\hline 147 & Popovac & 3,159 & 2,857 & 303 & 2,084 & 6.6 & 6.0 & 0.6 & 3 \\
\hline 148 & Podravske Sesvete & 3,144 & 3,432 & -288 & 1,630 & 5.1 & 5.6 & -0.5 & 3 \\
\hline 149 & Klinča Sela & 3,143 & 2,975 & 168 & 5,231 & 16.4 & 15.6 & 0.9 & 0 \\
\hline 150 & Trnava & 3,142 & 2,313 & 829 & 1,600 & 5.0 & 3.7 & 1.3 & 0 \\
\hline 151 & Jasenovac & 3,138 & 3,515 & -377 & 1,997 & 6.3 & 7.0 & -0.8 & 2 \\
\hline 152 & Semeljci & 3,131 & 2,592 & 540 & 4,362 & 13.7 & 11.3 & 2.4 & 2 \\
\hline 153 & Perušić & 3,118 & 3,521 & -403 & 2,638 & 8.2 & 9.3 & -1.1 & 3 \\
\hline 154 & Pučišća & 3,118 & 3,281 & -163 & 2,171 & 6.8 & 7.1 & -0.4 & 1 \\
\hline 155 & Posedarje & 3,112 & 3,298 & -187 & 3,607 & 11.2 & 11.9 & -0.7 & 1 \\
\hline 156 & Barilović & 3,082 & 2,666 & 416 & 2,990 & 9.2 & 8.0 & 1.2 & 3 \\
\hline 157 & Klis & 3,079 & 3,526 & -447 & 4,801 & 14.8 & 16.9 & -2.1 & 2 \\
\hline 158 & Gornji Kneginec & 3,078 & 1,710 & 1,368 & 5,349 & 16.5 & 9.1 & 7.3 & 3 \\
\hline 159 & Drnje & 3,067 & 2,728 & 338 & 1,863 & 5.7 & 5.1 & 0.6 & 4 \\
\hline 160 & Sukošan & 3,033 & 2,854 & 179 & 4,583 & 13.9 & 13.1 & 0.8 & 4 \\
\hline 161 & Lokvičići & 2,988 & 3,026 & -38 & 807 & 2.4 & 2.4 & 0.0 & 0 \\
\hline 162 & Stara Gradiška & 2,968 & 2,941 & 27 & 1,363 & 4.0 & 4.0 & 0.0 & 4 \\
\hline 163 & Novigrad & 2,952 & 4,530 & $-1,578$ & 2,375 & 7.0 & 10.8 & -3.7 & 2 \\
\hline
\end{tabular}


Per capita (in HRK)

Total (in million HRK)

\begin{tabular}{|c|c|c|c|c|c|c|c|c|c|}
\hline & Municipality & $\begin{array}{l}\text { Reve- } \\
\text { nues }\end{array}$ & $\begin{array}{l}\text { Expen- } \\
\text { ditures }\end{array}$ & $\begin{array}{l}\text { Surplus } \\
\text { /deficit }\end{array}$ & $\begin{array}{c}\text { Popula- } \\
\text { tion }\end{array}$ & $\begin{array}{l}\text { Reve- } \\
\text { nues }\end{array}$ & $\begin{array}{l}\text { Expen- } \\
\text { ditures }\end{array}$ & $\begin{array}{l}\text { Surplus } \\
\text { /deficit }\end{array}$ & $\begin{array}{l}\text { Trans- } \\
\text { paren- } \\
\text { cy }\end{array}$ \\
\hline 164 & Višnjan & 2,947 & 2,923 & 23 & 2,274 & 6.7 & 6.6 & 0.1 & 1 \\
\hline 165 & Brod Moravice & 2,936 & 2,841 & 95 & 866 & 2.5 & 2.5 & 0.1 & 2 \\
\hline 166 & $\begin{array}{l}\text { Novigrad } \\
\text { Podravski }\end{array}$ & 2,913 & 2,269 & 644 & 2,872 & 8.4 & 6.5 & 1.8 & 2 \\
\hline 167 & Vladislavci & 2,911 & 3,195 & -284 & 1,882 & 5.5 & 6.0 & -0.5 & 3 \\
\hline 168 & Seget & 2,910 & 2,717 & 193 & 4,854 & 14.1 & 13.2 & 0.9 & 3 \\
\hline 169 & Virje & 2,898 & 2,903 & -5 & 4,587 & 13.3 & 13.3 & 0.0 & 2 \\
\hline 170 & Jelenje & 2,890 & 3,491 & -601 & 5,344 & 15.4 & 18.7 & -3.2 & 0 \\
\hline 171 & Pušća & 2,883 & 3,385 & -502 & 2,700 & 7.8 & 9.1 & -1.4 & 2 \\
\hline 172 & Blato & 2,866 & 2,889 & -23 & 3,593 & 10.3 & 10.4 & -0.1 & 3 \\
\hline 173 & Darda & 2,854 & 2,287 & 567 & 6,908 & 19.7 & 15.8 & 3.9 & 0 \\
\hline 174 & Smokvica & 2,853 & 8,174 & $-5,321$ & 916 & 2.6 & 7.5 & -4.9 & 2 \\
\hline 175 & Bilje & 2,814 & 2,402 & 412 & 5,642 & 15.9 & 13.5 & 2.3 & 0 \\
\hline 176 & Satnica Đakovačka & 2,805 & 2,926 & -121 & 2,123 & 6.0 & 6.2 & -0.3 & 3 \\
\hline 177 & Ražanac & 2,800 & 2,650 & 150 & 2,940 & 8.2 & 7.8 & 0.4 & 1 \\
\hline 178 & Vuka & 2,782 & 3,649 & -866 & 1,200 & 3.3 & 4.4 & -1.0 & 4 \\
\hline 179 & Kapela & 2,758 & 2,235 & 523 & 2,984 & 8.2 & 6.7 & 1.6 & 2 \\
\hline 180 & Križ & 2,740 & 1,989 & 751 & 6,963 & 19.1 & 13.9 & 5.2 & 4 \\
\hline 181 & Lasinja & 2,731 & 2,730 & 0 & 1,624 & 4.4 & 4.4 & 0.0 & 3 \\
\hline 182 & Ružić & 2,722 & 2,627 & 95 & 1,591 & 4.3 & 4.2 & 0.2 & 4 \\
\hline 183 & Dekanovec & 2,717 & 2,875 & -158 & 774 & 2.1 & 2.2 & -0.1 & 4 \\
\hline 184 & Šestanovac & 2,701 & 2,243 & 459 & 1,958 & 5.3 & 4.4 & 0.9 & 1 \\
\hline 185 & Lekenik & 2,699 & 3,390 & -691 & 6,032 & 16.3 & 20.4 & -4.2 & 1 \\
\hline 186 & Gorjani & 2,696 & 2,330 & 366 & 1,591 & 4.3 & 3.7 & 0.6 & 0 \\
\hline 187 & Berek & 2,687 & 2,865 & -178 & 1,443 & 3.9 & 4.1 & -0.3 & 2 \\
\hline 188 & Jesenje & 2,683 & 3,247 & -563 & 1,560 & 4.2 & 5.1 & -0.9 & 2 \\
\hline 189 & Dicmo & 2,663 & 2,795 & -133 & 2,802 & 7.5 & 7.8 & -0.4 & 0 \\
\hline 190 & Sopje & 2,643 & 2,496 & 147 & 2,320 & 6.1 & 5.8 & 0.3 & 1 \\
\hline 191 & Dugi Rat & 2,633 & 2,366 & 267 & 7,092 & 18.7 & 16.8 & 1.9 & 0 \\
\hline 192 & Muć & 2,627 & 2,154 & 473 & 3,882 & 10.2 & 8.4 & 1.8 & 3 \\
\hline 193 & Gračac & 2,618 & 2,671 & -53 & 4,690 & 12.3 & 12.5 & -0.3 & 4 \\
\hline 194 & Cerovlje & 2,611 & 3,150 & -540 & 1,677 & 4.4 & 5.3 & -0.9 & 1 \\
\hline 195 & Tounj & 2,603 & 2,017 & 587 & 1,150 & 3.0 & 2.3 & 0.7 & 3 \\
\hline 196 & $\begin{array}{l}\text { Podravska } \\
\text { Moslavina }\end{array}$ & 2,569 & 2,845 & -276 & 1,202 & 3.1 & 3.4 & -0.3 & 0 \\
\hline 197 & Vrbanja & 2,542 & 2,541 & 1 & 3,940 & 10.0 & 10.0 & 0.0 & 2 \\
\hline 198 & Šodolovci & 2,536 & 2,792 & -256 & 1,653 & 4.2 & 4.6 & -0.4 & 3 \\
\hline 199 & Tompojevci & 2,526 & 2,569 & -43 & 1,565 & 4.0 & 4.0 & -0.1 & 3 \\
\hline 200 & Punitovci & 2,499 & 2,160 & 338 & 1,803 & 4.5 & 3.9 & 0.6 & 0 \\
\hline 201 & Hum na Sutli & 2,470 & 2,395 & 75 & 5,060 & 12.5 & 12.1 & 0.4 & 2 \\
\hline 202 & Voćin & 2,443 & 3,907 & $-1,465$ & 2,382 & 5.8 & 9.3 & -3.5 & 2 \\
\hline 203 & Šandrovac & 2,433 & 2,214 & 219 & 1,776 & 4.3 & 3.9 & 0.4 & 2 \\
\hline 204 & Vela Luka & 2,407 & 2,256 & 151 & 4,137 & 10.0 & 9.3 & 0.6 & 0 \\
\hline 205 & Radoboj & 2,406 & 2,733 & -328 & 3,387 & 8.1 & 9.3 & -1.1 & 5 \\
\hline 206 & Zagorska Sela & 2,404 & 2,496 & -92 & 996 & 2.4 & 2.5 & -0.1 & 0 \\
\hline 207 & Brdovec & 2,403 & 2,735 & -332 & 11,134 & 26.8 & 30.5 & -3.7 & 3 \\
\hline 208 & Žminj & 2,387 & 2,221 & 166 & 3,483 & 8.3 & 7.7 & 0.6 & 3 \\
\hline 209 & Škabrnja & 2,383 & 2,399 & -15 & 1,776 & 4.2 & 4.3 & 0.0 & 0 \\
\hline 210 & Privlaka (Vinkovci) & 2,364 & 1,868 & 496 & 2,954 & 7.0 & 5.5 & 1.5 & 1 \\
\hline 211 & Jakšić & 2,360 & 1,415 & 945 & 4,058 & 9.6 & 5.7 & 3.8 & 1 \\
\hline 212 & Vojnić & 2,352 & 2,035 & 317 & 4,764 & 11.2 & 9.7 & 1.5 & 3 \\
\hline 213 & Feričanci & 2,349 & 2,182 & 167 & 2,134 & 5.0 & 4.7 & 0.4 & 3 \\
\hline 214 & Zmijavci & 2,345 & 1,878 & 467 & 2,048 & 4.8 & 3.8 & 1.0 & 0 \\
\hline 215 & Topusko & 2,345 & 2,251 & 93 & 2,985 & 7.0 & 6.7 & 0.3 & 3 \\
\hline 216 & Oriovac & 2,332 & 2,609 & -277 & 5,824 & 13.6 & 15.2 & -1.6 & 1 \\
\hline 217 & Erdut & 2,331 & 2,462 & -131 & 7,308 & 17.0 & 18.0 & -1.0 & 3 \\
\hline 218 & Klenovnik & 2,328 & 1,800 & 529 & 2,022 & 4.7 & 3.6 & 1.1 & 0 \\
\hline
\end{tabular}

NEWSLETTER Io8 | K. OTT, M. BRONIĆ, B. STANIĆ | Budget outturns of Croatian municipalities... | Institute of Public Finance 7 
Per capita (in HRK)

Total (in million HRK)

\begin{tabular}{|c|c|c|c|c|c|c|c|c|c|}
\hline & Municipality & $\begin{array}{l}\text { Reve- } \\
\text { nues }\end{array}$ & $\begin{array}{l}\text { Expen- } \\
\text { ditures }\end{array}$ & $\begin{array}{l}\text { Surplus } \\
\text { /deficit }\end{array}$ & $\begin{array}{l}\text { Popula- } \\
\text { tion }\end{array}$ & $\begin{array}{l}\text { Reve- } \\
\text { nues }\end{array}$ & $\begin{array}{l}\text { Expen- } \\
\text { ditures }\end{array}$ & $\begin{array}{l}\text { Surplus } \\
\text { /deficit }\end{array}$ & $\begin{array}{l}\text { Trans- } \\
\text { paren- } \\
\text { cy }\end{array}$ \\
\hline 219 & Žumberak & 2,325 & 2,443 & -118 & 883 & 2.1 & 2.2 & -0.1 & 0 \\
\hline 220 & Otok (Split) & 2,319 & 2,482 & -163 & 5,474 & 12.7 & 13.6 & -0.9 & 1 \\
\hline 221 & Crnac & 2,303 & 2,974 & -672 & 1,456 & 3.4 & 4.3 & -1.0 & 3 \\
\hline 222 & Hlebine & 2,303 & 2,492 & -189 & 1,304 & 3.0 & 3.2 & -0.2 & 4 \\
\hline 223 & Bogdanovci & 2,302 & 2,369 & -67 & 1,960 & 4.5 & 4.6 & -0.1 & 1 \\
\hline 224 & Ernestinovo & 2,290 & 2,138 & 152 & 2,189 & 5.0 & 4.7 & 0.3 & 3 \\
\hline 225 & Stari Jankovci & 2,266 & 2,282 & -15 & 4,405 & 10.0 & 10.1 & -0.1 & 4 \\
\hline 226 & Bilice & 2,264 & 2,950 & -685 & 2,307 & 5.2 & 6.8 & -1.6 & 1 \\
\hline 227 & Koprivnički Ivanec & 2,258 & 2,915 & -657 & 2,121 & 4.8 & 6.2 & -1.4 & 2 \\
\hline 228 & Biskupija & 2,247 & 2,429 & -182 & 1,699 & 3.8 & 4.1 & -0.3 & 2 \\
\hline 229 & Velika Trnovitica & 2,241 & 2,121 & 120 & 1,370 & 3.1 & 2.9 & 0.2 & 1 \\
\hline 230 & Vrhovine & 2,235 & 2,529 & -294 & 1,381 & 3.1 & 3.5 & -0.4 & 2 \\
\hline 231 & Novo Virje & 2,215 & 1,975 & 240 & 1,216 & 2.7 & 2.4 & 0.3 & 3 \\
\hline 232 & Lipovljani & 2,212 & 2,521 & -309 & 3,455 & 7.6 & 8.7 & -1.1 & 4 \\
\hline 233 & Tordinci & 2,209 & 2,285 & -76 & 2,032 & 4.5 & 4.6 & -0.2 & 0 \\
\hline 234 & Čađavica & 2,204 & 2,780 & -576 & 2,009 & 4.4 & 5.6 & -1.2 & 3 \\
\hline 235 & Josipdol & 2,166 & 2,188 & -22 & 3,773 & 8.2 & 8.3 & -0.1 & 4 \\
\hline 236 & Marija Bistrica & 2,157 & 2,147 & 11 & 5,976 & 12.9 & 12.8 & 0.1 & 0 \\
\hline 237 & Veliko Trojstvo & 2,154 & 1,982 & 172 & 2,741 & 5.9 & 5.4 & 0.5 & 1 \\
\hline 238 & Žakanje & 2,147 & 1,769 & 378 & 1,889 & 4.1 & 3.3 & 0.7 & 2 \\
\hline 239 & Kravarsko & 2,119 & 2,405 & -286 & 1,987 & 4.2 & 4.8 & -0.6 & 2 \\
\hline 240 & Hercegovac & 2,115 & 1,652 & 462 & 2,383 & 5.0 & 3.9 & 1.1 & 3 \\
\hline 241 & Veliko Trgovišće & 2,114 & 2,163 & -49 & 4,945 & 10.5 & 10.7 & -0.2 & 2 \\
\hline 242 & Pokupsko & 2,106 & 5,629 & $-3,524$ & 2,224 & 4.7 & 12.5 & -7.8 & 1 \\
\hline 243 & Krnjak & 2,099 & 1,912 & 187 & 1,985 & 4.2 & 3.8 & 0.4 & 2 \\
\hline 244 & Ivanska & 2,096 & 2,095 & 1 & 2,911 & 6.1 & 6.1 & 0.0 & 1 \\
\hline 245 & Bizovac & 2,092 & 1,992 & 100 & 4,507 & 9.4 & 9.0 & 0.4 & 5 \\
\hline 246 & Štefanje & 2,085 & 2,073 & 12 & 2,030 & 4.2 & 4.2 & 0.0 & 0 \\
\hline 247 & Rugvica & 2,084 & 2,143 & -59 & 7,871 & 16.4 & 16.9 & -0.5 & 0 \\
\hline 248 & Zlatar Bistrica & 2,081 & 2,140 & -59 & 2,600 & 5.4 & 5.6 & -0.2 & 1 \\
\hline 249 & Cista Provo & 2,069 & 2,264 & -195 & 2,335 & 4.8 & 5.3 & -0.5 & 1 \\
\hline 250 & Unešić & 2,056 & 2,827 & -770 & 1,686 & 3.5 & 4.8 & -1.3 & 3 \\
\hline 251 & Slivno & 2,056 & 1,887 & 170 & 1,999 & 4.1 & 3.8 & 0.3 & 2 \\
\hline 252 & Kotoriba & 2,039 & 2,074 & -35 & 3,224 & 6.6 & 6.7 & -0.1 & 3 \\
\hline 253 & Petrijevci & 2,037 & 1,769 & 268 & 2,870 & 5.8 & 5.1 & 0.8 & 3 \\
\hline 254 & Tuhelj & 2,027 & 2,460 & -433 & 2,104 & 4.3 & 5.2 & -0.9 & 2 \\
\hline 255 & Brinje & 2,018 & 2,208 & -190 & 3,256 & 6.6 & 7.2 & -0.6 & 3 \\
\hline 256 & Šenkovec & 2,016 & 1,839 & 177 & 2,879 & 5.8 & 5.3 & 0.5 & 2 \\
\hline 257 & Pitomača & 2,014 & 1,831 & 183 & 10,059 & 20.3 & 18.4 & 1.8 & 4 \\
\hline 258 & Viljevo & 2,011 & 2,011 & 1 & 2,065 & 4.2 & 4.2 & 0.0 & 2 \\
\hline 259 & Sveti Ilija & 2,006 & 2,228 & -221 & 3,511 & 7.0 & 7.8 & -0.8 & 1 \\
\hline 260 & Brestovac & 1,997 & 2,049 & -53 & 3,726 & 7.4 & 7.6 & -0.2 & 3 \\
\hline 261 & Nova Kapela & 1,971 & 1,655 & 317 & 4,227 & 8.3 & 7.0 & 1.3 & 0 \\
\hline 262 & Cestica & 1,957 & 2,248 & -291 & 5,806 & 11.4 & 13.1 & -1.7 & 3 \\
\hline 263 & Kloštar Ivanić & 1,952 & 2,470 & -518 & 6,091 & 11.9 & 15.0 & -3.2 & 4 \\
\hline 264 & Karojba & 1,949 & 2,022 & -73 & 1,438 & 2.8 & 2.9 & -0.1 & 2 \\
\hline 265 & Kloštar Podravski & 1,940 & 1,834 & 106 & 3,306 & 6.4 & 6.1 & 0.3 & 0 \\
\hline 266 & Babina Greda & 1,932 & 1,564 & 368 & 3,572 & 6.9 & 5.6 & 1.3 & 4 \\
\hline 267 & Đurmanec & 1,924 & 1,995 & -71 & 4,235 & 8.1 & 8.5 & -0.3 & 1 \\
\hline 268 & Velika Ludina & 1,922 & 2,677 & -755 & 2,625 & 5.0 & 7.0 & -2.0 & 2 \\
\hline 269 & Gradina & 1,921 & 2,129 & -208 & 3,850 & 7.4 & 8.2 & -0.8 & 3 \\
\hline 270 & Lukač & 1,915 & 1,928 & -13 & 3,634 & 7.0 & 7.0 & 0.0 & 3 \\
\hline 271 & Donji Kukuruzari & 1,912 & 1,891 & 21 & 1,634 & 3.1 & 3.1 & 0.0 & 2 \\
\hline 272 & Podgorač & 1,912 & 1,493 & 419 & 2,877 & 5.5 & 4.3 & 1.2 & 3 \\
\hline 273 & $\begin{array}{l}\text { Trnovec } \\
\text { Bartolovečki }\end{array}$ & 1,909 & 1,889 & 20 & 6,884 & 13.1 & 13.0 & 0.1 & 3 \\
\hline
\end{tabular}


Per capita (in HRK)

Total (in million HRK)

\begin{tabular}{|c|c|c|c|c|c|c|c|c|c|}
\hline & Municipality & $\begin{array}{l}\text { Reve- } \\
\text { nues }\end{array}$ & $\begin{array}{l}\text { Expen- } \\
\text { ditures }\end{array}$ & $\begin{array}{l}\text { Surplus } \\
\text { /deficit }\end{array}$ & $\begin{array}{l}\text { Popula- } \\
\text { tion }\end{array}$ & $\begin{array}{l}\text { Reve- } \\
\text { nues }\end{array}$ & $\begin{array}{l}\text { Expen- } \\
\text { ditures }\end{array}$ & $\begin{array}{l}\text { Surplus } \\
\text { /deficit }\end{array}$ & $\begin{array}{l}\text { Trans- } \\
\text { paren- } \\
\text { cy }\end{array}$ \\
\hline 274 & Plaški & 1,903 & 1,700 & 203 & 2,090 & 4.0 & 3.6 & 0.4 & 0 \\
\hline 275 & Bosiljevo & 1,897 & 2,145 & -248 & 1,284 & 2.4 & 2.8 & -0.3 & 2 \\
\hline 276 & Sračinec & 1,897 & 1,853 & 44 & 4,842 & 9.2 & 9.0 & 0.2 & 2 \\
\hline 277 & Bošnjaci & 1,893 & 1,933 & -40 & 3,901 & 7.4 & 7.5 & -0.2 & 1 \\
\hline 278 & $\begin{array}{l}\text { Sveti Martin } \\
\text { na Muri }\end{array}$ & 1,887 & 1,565 & 322 & 2,605 & 4.9 & 4.1 & 0.8 & 3 \\
\hline 279 & Peteranec & 1,877 & 1,470 & 407 & 2,704 & 5.1 & 4.0 & 1.1 & 2 \\
\hline 280 & Breznica & 1,874 & 1,025 & 849 & 2,200 & 4.1 & 2.3 & 1.9 & 0 \\
\hline 281 & Čačinci & 1,865 & 1,794 & 71 & 2,802 & 5.2 & 5.0 & 0.2 & 1 \\
\hline 282 & Klakar & 1,858 & 1,920 & -62 & 2,319 & 4.3 & 4.5 & -0.1 & 3 \\
\hline 283 & Runovići & 1,850 & 1,861 & -11 & 2,416 & 4.5 & 4.5 & 0.0 & 0 \\
\hline 284 & Severin & 1,838 & 1,710 & 128 & 877 & 1.6 & 1.5 & 0.1 & 0 \\
\hline 285 & Generalski Stol & 1,835 & 2,020 & -184 & 2,642 & 4.8 & 5.3 & -0.5 & 4 \\
\hline 286 & Podbablje & 1,834 & 1,396 & 437 & 4,680 & 8.6 & 6.5 & 2.0 & 0 \\
\hline 287 & Dubravica & 1,830 & 2,115 & -285 & 1,437 & 2.6 & 3.0 & -0.4 & 1 \\
\hline 288 & Konjščina & 1,827 & 1,813 & 14 & 3,790 & 6.9 & 6.9 & 0.1 & 4 \\
\hline 289 & Kraljevec na Sutli & 1,826 & 1,928 & -102 & 1,727 & 3.2 & 3.3 & -0.2 & 0 \\
\hline 290 & Belica & 1,826 & 1,588 & 238 & 3,176 & 5.8 & 5.0 & 0.8 & 4 \\
\hline 291 & Zdenci & 1,824 & 1,926 & -102 & 1,904 & 3.5 & 3.7 & -0.2 & 1 \\
\hline 292 & Velika Pisanica & 1,821 & 1,618 & 204 & 1,781 & 3.2 & 2.9 & 0.4 & 1 \\
\hline 293 & Brodski Stupnik & 1,821 & 1,262 & 559 & 3,036 & 5.5 & 3.8 & 1.7 & 4 \\
\hline 294 & Marijanci & 1,820 & 1,165 & 655 & 2,405 & 4.4 & 2.8 & 1.6 & 1 \\
\hline 295 & Koška & 1,816 & 1,390 & 426 & 3,980 & 7.2 & 5.5 & 1.7 & 3 \\
\hline 296 & Kistanje & 1,807 & 1,688 & 119 & 3,481 & 6.3 & 5.9 & 0.4 & 5 \\
\hline 297 & Nova Bukovica & 1,802 & 2,497 & -695 & 1,771 & 3.2 & 4.4 & -1.2 & 1 \\
\hline 298 & Lovreć & 1,801 & 1,700 & 101 & 1,699 & 3.1 & 2.9 & 0.2 & 1 \\
\hline 299 & Brckovljani & 1,800 & 1,572 & 228 & 6,837 & 12.3 & 10.7 & 1.6 & 0 \\
\hline 300 & Domašinec & 1,795 & 1,436 & 359 & 2,251 & 4.0 & 3.2 & 0.8 & 2 \\
\hline 301 & Bedenica & 1,790 & 2,205 & -416 & 1,432 & 2.6 & 3.2 & -0.6 & 0 \\
\hline 302 & Rakovec & 1,788 & 1,759 & 29 & 1,252 & 2.2 & 2.2 & 0.0 & 3 \\
\hline 303 & Stankovci & 1,787 & 2,822 & $-1,035$ & 2,003 & 3.6 & 5.7 & -2.1 & 0 \\
\hline 304 & Orehovica & 1,780 & 1,646 & 134 & 2,685 & 4.8 & 4.4 & 0.4 & 5 \\
\hline 305 & Gornji Bogićevci & 1,779 & 1,558 & 221 & 1,975 & 3.5 & 3.1 & 0.4 & 0 \\
\hline 306 & Bednja & 1,763 & 1,650 & 113 & 3,992 & 7.0 & 6.6 & 0.5 & 2 \\
\hline 307 & Donji Lapac & 1,728 & 1,945 & -217 & 2,113 & 3.7 & 4.1 & -0.5 & 1 \\
\hline 308 & Gornji Mihaljevec & 1,725 & 1,468 & 258 & 1,917 & 3.3 & 2.8 & 0.5 & 3 \\
\hline 309 & Martinska Ves & 1,725 & 1,248 & 477 & 3,488 & 6.0 & 4.4 & 1.7 & 3 \\
\hline 310 & Jakovlje & 1,722 & 1,502 & 220 & 3,930 & 6.8 & 5.9 & 0.9 & 0 \\
\hline 311 & Netretić & 1,720 & 1,848 & -128 & 2,862 & 4.9 & 5.3 & -0.4 & 4 \\
\hline 312 & Vinica & 1,716 & 1,634 & 82 & 3,389 & 5.8 & 5.5 & 0.3 & 2 \\
\hline 313 & Krapinske Toplice & 1,710 & 1,809 & -99 & 5,367 & 9.2 & 9.7 & -0.5 & 4 \\
\hline 314 & Breznički Hum & 1,700 & 1,508 & 193 & 1,356 & 2.3 & 2.0 & 0.3 & 3 \\
\hline 315 & Selnica & 1,699 & 1,698 & 1 & 2,991 & 5.1 & 5.1 & 0.0 & 2 \\
\hline 316 & Novi Golubovec & 1,698 & 1,805 & -107 & 996 & 1.7 & 1.8 & -0.1 & 0 \\
\hline 317 & Gornja Stubica & 1,690 & 1,733 & -43 & 5,284 & 8.9 & 9.2 & -0.2 & 0 \\
\hline 318 & Staro Petrovo Selo & 1,679 & 1,347 & 333 & 5,186 & 8.7 & 7.0 & 1.7 & 3 \\
\hline 319 & Nuštar & 1,677 & 1,743 & -66 & 5,793 & 9.7 & 10.1 & -0.4 & 3 \\
\hline 320 & Ferdinandovac & 1,675 & 1,678 & -3 & 1,750 & 2.9 & 2.9 & 0.0 & 3 \\
\hline 321 & Kumrovec & 1,662 & 1,871 & -210 & 1,588 & 2.6 & 3.0 & -0.3 & 1 \\
\hline 322 & Pribislavec & 1,652 & 1,651 & 1 & 3,136 & 5.2 & 5.2 & 0.0 & 2 \\
\hline 323 & Vrpolje & 1,646 & 1,203 & 443 & 3,521 & 5.8 & 4.2 & 1.6 & 3 \\
\hline 324 & Orle & 1,646 & 1,653 & -7 & 1,975 & 3.3 & 3.3 & 0.0 & 0 \\
\hline 325 & Gornja Vrba & 1,646 & 1,449 & 196 & 2,512 & 4.1 & 3.6 & 0.5 & 3 \\
\hline 326 & Nedelišće & 1,642 & 1,591 & 51 & 11,975 & 19.7 & 19.1 & 0.6 & 1 \\
\hline 327 & Mihovljan & 1,638 & 1,393 & 245 & 1,938 & 3.2 & 2.7 & 0.5 & 0 \\
\hline 328 & Levanjska Varoš & 1,631 & 1,532 & 99 & 1,194 & 1.9 & 1.8 & 0.1 & 0 \\
\hline
\end{tabular}

NEWSLETTER IO8 | K. OTT, M. BRONIĆ, B. STANIĆ | Budget outturns of Croatian municipalities... | Institute of Public Finance 9 
Per capita (in HRK)

Total (in million HRK)

\begin{tabular}{|c|c|c|c|c|c|c|c|c|c|}
\hline & Municipality & $\begin{array}{l}\text { Reve- } \\
\text { nues }\end{array}$ & $\begin{array}{l}\text { Expen- } \\
\text { ditures }\end{array}$ & $\begin{array}{l}\text { Surplus } \\
\text { /deficit }\end{array}$ & $\begin{array}{c}\text { Popula- } \\
\text { tion }\end{array}$ & $\begin{array}{l}\text { Reve- } \\
\text { nues }\end{array}$ & $\begin{array}{l}\text { Expen- } \\
\text { ditures }\end{array}$ & $\begin{array}{l}\text { Surplus } \\
\text { /deficit }\end{array}$ & $\begin{array}{l}\text { Trans- } \\
\text { paren- } \\
\text { cy }\end{array}$ \\
\hline 329 & Hrvatska Dubica & 1,615 & 1,721 & -105 & 2,089 & 3.4 & 3.6 & -0.2 & 3 \\
\hline 330 & Gundinci & 1,609 & 1,097 & 512 & 2,027 & 3.3 & 2.2 & 1.0 & 2 \\
\hline 331 & Čepin & 1,604 & 1,402 & 202 & 11,599 & 18.6 & 16.3 & 2.3 & 0 \\
\hline 332 & Cerna & 1,603 & 1,362 & 242 & 4,595 & 7.4 & 6.3 & 1.1 & 1 \\
\hline 333 & Galovac & 1,603 & 1,934 & -331 & 1,234 & 2.0 & 2.4 & -0.4 & 1 \\
\hline 334 & Dragalić & 1,593 & 1,694 & -101 & 1,361 & 2.2 & 2.3 & -0.1 & 2 \\
\hline 335 & Okučani & 1,587 & 1,564 & 23 & 3,447 & 5.5 & 5.4 & 0.1 & 1 \\
\hline 336 & Bukovlje & 1,583 & 1,244 & 339 & 3,108 & 4.9 & 3.9 & 1.1 & 4 \\
\hline 337 & Gradec & 1,576 & 1,553 & 22 & 3,681 & 5.8 & 5.7 & 0.1 & 2 \\
\hline 338 & Donja Motičina & 1,574 & 1,072 & 503 & 1,652 & 2.6 & 1.8 & 0.8 & 2 \\
\hline 339 & Štrigova & 1,574 & 1,620 & -46 & 2,766 & 4.4 & 4.5 & -0.1 & 2 \\
\hline 340 & Kula Norinska & 1,565 & 1,571 & -6 & 1,748 & 2.7 & 2.7 & 0.0 & 2 \\
\hline 341 & Negoslavci & 1,561 & 1,451 & 110 & 1,463 & 2.3 & 2.1 & 0.2 & 1 \\
\hline 342 & Đulovac & 1,553 & 1,399 & 154 & 3,245 & 5.0 & 4.5 & 0.5 & 4 \\
\hline 343 & Draganić & 1,552 & 1,605 & -52 & 2,741 & 4.3 & 4.4 & -0.1 & 3 \\
\hline 344 & Andrijaševci & 1,548 & 1,686 & -138 & 4,075 & 6.3 & 6.9 & -0.6 & 2 \\
\hline 345 & Donja Dubrava & 1,544 & 1,604 & -61 & 1,920 & 3.0 & 3.1 & -0.1 & 2 \\
\hline 346 & Trpinja & 1,542 & 1,634 & -92 & 5,572 & 8.6 & 9.1 & -0.5 & 3 \\
\hline 347 & $\begin{array}{l}\text { Sveti Petar } \\
\text { Orehovec }\end{array}$ & 1,540 & 983 & 557 & 4,583 & 7.1 & 4.5 & 2.6 & 2 \\
\hline 348 & Hrašćina & 1,536 & 1,462 & 74 & 1,617 & 2.5 & 2.4 & 0.1 & 0 \\
\hline 349 & Vođinci & 1,536 & 1,701 & -166 & 1,966 & 3.0 & 3.3 & -0.3 & 3 \\
\hline 350 & Petrovsko & 1,535 & 1,260 & 276 & 2,656 & 4.1 & 3.3 & 0.7 & 1 \\
\hline 351 & Cernik & 1,530 & 1,420 & 110 & 3,640 & 5.6 & 5.2 & 0.4 & 3 \\
\hline 352 & Zrinski Topolovac & 1,525 & 1,735 & -210 & 890 & 1.4 & 1.5 & -0.2 & 0 \\
\hline 353 & Velika & 1,524 & 1,333 & 192 & 5,607 & 8.5 & 7.5 & 1.1 & 3 \\
\hline 354 & Viškovci & 1,522 & 1,639 & -118 & 1,906 & 2.9 & 3.1 & -0.2 & 0 \\
\hline 355 & Velika Kopanica & 1,518 & 1,501 & 17 & 3,308 & 5.0 & 5.0 & 0.1 & 0 \\
\hline 356 & Donji Andrijevci & 1,518 & 1,593 & -75 & 3,709 & 5.6 & 5.9 & -0.3 & 3 \\
\hline 357 & Đurđenovac & 1,515 & 1,192 & 323 & 6,750 & 10.2 & 8.0 & 2.2 & 0 \\
\hline 358 & Mala Subotica & 1,512 & 1,074 & 438 & 5,452 & 8.2 & 5.9 & 2.4 & 3 \\
\hline 359 & Ivankovo & 1,505 & 1,246 & 259 & 8,006 & 12.1 & 10.0 & 2.1 & 1 \\
\hline 360 & Sunja & 1,503 & 1,694 & -190 & 5,748 & 8.6 & 9.7 & -1.1 & 4 \\
\hline 361 & Garčin & 1,503 & 1,557 & -54 & 4,806 & 7.2 & 7.5 & -0.3 & 3 \\
\hline 362 & Rasinja & 1,497 & 1,461 & 36 & 3,267 & 4.9 & 4.8 & 0.1 & 0 \\
\hline 363 & Cetingrad & 1,496 & 1,577 & -81 & 2,027 & 3.0 & 3.2 & -0.2 & 2 \\
\hline 364 & Beretinec & 1,478 & 1,435 & 43 & 2,176 & 3.2 & 3.1 & 0.1 & 3 \\
\hline 365 & Dubrava & 1,472 & 1,475 & -2 & 5,245 & 7.7 & 7.7 & 0.0 & 2 \\
\hline 366 & Dežanovac & 1,471 & 2,238 & -767 & 2,715 & 4.0 & 6.1 & -2.1 & 2 \\
\hline 367 & Markušica & 1,469 & 1,542 & -73 & 2,555 & 3.8 & 3.9 & -0.2 & 2 \\
\hline 368 & Preseka & 1,465 & 1,237 & 227 & 1,448 & 2.1 & 1.8 & 0.3 & 1 \\
\hline 369 & Oprisavci & 1,462 & 1,534 & -72 & 2,508 & 3.7 & 3.8 & -0.2 & 2 \\
\hline 370 & Vratišinec & 1,458 & 1,399 & 60 & 1,984 & 2.9 & 2.8 & 0.1 & 2 \\
\hline 371 & Sveti Križ Začretje & 1,457 & 1,356 & 101 & 6,165 & 9.0 & 8.4 & 0.6 & 5 \\
\hline 372 & Mikleuš & 1,457 & 1,670 & -213 & 1,464 & 2.1 & 2.4 & -0.3 & 0 \\
\hline 373 & Suhopolje & 1,456 & 1,710 & -255 & 6,683 & 9.7 & 11.4 & -1.7 & 3 \\
\hline 374 & Stari Mikanovci & 1,452 & 1,416 & 35 & 2,956 & 4.3 & 4.2 & 0.1 & 2 \\
\hline 375 & Strahoninec & 1,452 & 1,049 & 402 & 2,682 & 3.9 & 2.8 & 1.1 & 4 \\
\hline 376 & Veliki Bukovec & 1,449 & 1,223 & 226 & 1,438 & 2.1 & 1.8 & 0.3 & 1 \\
\hline 377 & Gradište & 1,448 & 1,464 & -16 & 2,773 & 4.0 & 4.1 & 0.0 & 1 \\
\hline 378 & Gvozd & 1,437 & 1,328 & 109 & 2,970 & 4.3 & 3.9 & 0.3 & 1 \\
\hline 379 & Sveti Đurd & 1,430 & 1,401 & 29 & 3,804 & 5.4 & 5.3 & 0.1 & 1 \\
\hline 380 & Mali Bukovec & 1,425 & 1,270 & 155 & 2,212 & 3.2 & 2.8 & 0.3 & 3 \\
\hline 381 & Čaglin & 1,410 & 1,587 & -177 & 2,723 & 3.8 & 4.3 & -0.5 & 2 \\
\hline 382 & Dvor & 1,408 & 1,395 & 12 & 5,570 & 7.8 & 7.8 & 0.1 & 1 \\
\hline 383 & Rešetari & 1,401 & 1,344 & 57 & 4,753 & 6.7 & 6.4 & 0.3 & 1 \\
\hline
\end{tabular}

IO NEWSLETTER IO8 | K. OTT, M. BRONIĆ, B. STANIĆ | Budget outturns of Croatian municipalities... | Institute of Public Finance 
Per capita (in HRK)

Total (in million HRK)

\begin{tabular}{|c|c|c|c|c|c|c|c|c|c|}
\hline & Municipality & $\begin{array}{c}\text { Reve- } \\
\text { nues }\end{array}$ & $\begin{array}{l}\text { Expen- } \\
\text { ditures }\end{array}$ & $\begin{array}{l}\text { Surplus } \\
\text { /deficit }\end{array}$ & $\begin{array}{c}\text { Popula- } \\
\text { tion }\end{array}$ & $\begin{array}{c}\text { Reve- } \\
\text { nues }\end{array}$ & $\begin{array}{l}\text { Expen- } \\
\text { ditures }\end{array}$ & $\begin{array}{l}\text { Surplus } \\
\text { /deficit }\end{array}$ & $\begin{array}{l}\text { Trans- } \\
\text { paren- } \\
\text { cy }\end{array}$ \\
\hline 384 & Proložac & 1,398 & 1,678 & -281 & 3,802 & 5.3 & 6.4 & -1.1 & 0 \\
\hline 385 & Vrbje & 1,391 & 1,252 & 140 & 2,215 & 3.1 & 2.8 & 0.3 & 1 \\
\hline 386 & Sveta Marija & 1,370 & 1,603 & -232 & 2,317 & 3.2 & 3.7 & -0.5 & 3 \\
\hline 387 & Budinščina & 1,361 & 1,265 & 96 & 2,503 & 3.4 & 3.2 & 0.2 & 2 \\
\hline 388 & Nova Rača & 1,346 & 1,334 & 12 & 3,433 & 4.6 & 4.6 & 0.0 & 0 \\
\hline 389 & Martijanec & 1,345 & 1,242 & 103 & 3,843 & 5.2 & 4.8 & 0.4 & 4 \\
\hline 390 & Sveti Ivan Žabno & 1,340 & 1,048 & 291 & 5,222 & 7.0 & 5.5 & 1.5 & 2 \\
\hline 391 & Rovišće & 1,333 & 1,139 & 194 & 4,822 & 6.4 & 5.5 & 0.9 & 0 \\
\hline 392 & Maruševec & 1,323 & 1,013 & 310 & 6,381 & 8.4 & 6.5 & 2.0 & 3 \\
\hline 393 & Končanica & 1,322 & 1,344 & -22 & 2,360 & 3.1 & 3.2 & -0.1 & 2 \\
\hline 394 & Kaptol & 1,314 & 2,523 & $-1,209$ & 3,472 & 4.6 & 8.8 & -4.2 & 2 \\
\hline 395 & Podturen & 1,310 & 1,193 & 117 & 3,873 & 5.1 & 4.6 & 0.5 & 3 \\
\hline 396 & Gornja Rijeka & 1,309 & 1,772 & -463 & 1,779 & 2.3 & 3.2 & -0.8 & 1 \\
\hline 397 & Petrijanec & 1,302 & 1,372 & -70 & 4,812 & 6.3 & 6.6 & -0.3 & 1 \\
\hline 398 & Đelekovec & 1,296 & 1,294 & 2 & 1,533 & 2.0 & 2.0 & 0.0 & 3 \\
\hline 399 & Koprivnički Bregi & 1,294 & 1,134 & 160 & 2,381 & 3.1 & 2.7 & 0.4 & 3 \\
\hline 400 & Farkaševac & 1,292 & 1,379 & -87 & 1,937 & 2.5 & 2.7 & -0.2 & 1 \\
\hline 401 & Jalžabet & 1,288 & 3,057 & $-1,769$ & 3,615 & 4.7 & 11.0 & -6.4 & 0 \\
\hline 402 & Slavonski Šamac & 1,287 & 1,343 & -56 & 2,169 & 2.8 & 2.9 & -0.1 & 3 \\
\hline 403 & Špišić Bukovica & 1,284 & 1,334 & -50 & 4,221 & 5.4 & 5.6 & -0.2 & 0 \\
\hline 404 & Sibinj & 1,284 & 1,037 & 246 & 6,895 & 8.8 & 7.2 & 1.7 & 0 \\
\hline 405 & Bedekovčina & 1,283 & 1,263 & 20 & 8,041 & 10.3 & 10.2 & 0.2 & 4 \\
\hline 406 & Lobor & 1,282 & 1,301 & -19 & 3,188 & 4.1 & 4.1 & -0.1 & 3 \\
\hline 407 & Davor & 1,281 & 1,382 & -101 & 3,015 & 3.9 & 4.2 & -0.3 & 1 \\
\hline 408 & Desinić & 1,271 & 1,073 & 198 & 2,933 & 3.7 & 3.1 & 0.6 & 3 \\
\hline 409 & Drenje & 1,249 & 974 & 274 & 2,700 & 3.4 & 2.6 & 0.7 & 2 \\
\hline 410 & Donji Kraljevec & 1,248 & 1,154 & 94 & 4,659 & 5.8 & 5.4 & 0.4 & 3 \\
\hline 411 & Donji Vidovec & 1,224 & 1,550 & -326 & 1,399 & 1.7 & 2.2 & -0.5 & 2 \\
\hline 412 & Goričan & 1,213 & 1,211 & 2 & 2,823 & 3.4 & 3.4 & 0.0 & 3 \\
\hline 413 & Vidovec & 1,209 & 1,254 & -44 & 5,425 & 6.6 & 6.8 & -0.2 & 2 \\
\hline 414 & Jarmina & 1,180 & 1,328 & -148 & 2,458 & 2.9 & 3.3 & -0.4 & 3 \\
\hline 415 & Podcrkavlje & 1,177 & 1,308 & -131 & 2,553 & 3.0 & 3.3 & -0.3 & 3 \\
\hline 416 & Visoko & 1,167 & 869 & 299 & 1,518 & 1.8 & 1.3 & 0.5 & 0 \\
\hline 417 & Krašić & 1,160 & 1,295 & -135 & 2,640 & 3.1 & 3.4 & -0.4 & 3 \\
\hline 418 & $\begin{array}{l}\text { Sveti Juraj } \\
\text { na Bregu }\end{array}$ & 1,156 & 1,632 & -476 & 5,090 & 5.9 & 8.3 & -2.4 & 2 \\
\hline 419 & Bebrina & 1,108 & 975 & 133 & 3,252 & 3.6 & 3.2 & 0.4 & 4 \\
\hline 420 & Zažablje & 1,096 & 1,046 & 50 & 757 & 0.8 & 0.8 & 0.0 & 0 \\
\hline 421 & Donja Voća & 1,088 & 877 & 211 & 2,443 & 2.7 & 2.1 & 0.5 & 1 \\
\hline 422 & Sokolovac & 1,083 & 1,023 & 60 & 3,417 & 3.7 & 3.5 & 0.2 & 2 \\
\hline 423 & Štitar & 1,030 & 894 & 136 & 2,129 & 2.2 & 1.9 & 0.3 & 3 \\
\hline 424 & Borovo & 1,029 & 1,252 & -223 & 5,056 & 5.2 & 6.3 & -1.1 & 3 \\
\hline 425 & Sikirevci & 994 & 1,120 & -126 & 2,476 & 2.5 & 2.8 & -0.3 & 1 \\
\hline 426 & Strizivojna & 994 & 670 & 324 & 2,525 & 2.5 & 1.7 & 0.8 & 1 \\
\hline 427 & Mače & 960 & 855 & 105 & 2,534 & 2.4 & 2.2 & 0.3 & 3 \\
\hline 428 & Pojezerje & 954 & 2,020 & $-1,066$ & 991 & 0.9 & 2.0 & -1.1 & 0 \\
\hline
\end{tabular}

Source: Authors' data based on: Ministry of Finance (20I6), CBS (2016) and Ott et al. (2016). 
Table 2.

Budget outturns of cities, 2015.

Per capita (in HRK)

Total (in million HRK)

\begin{tabular}{|c|c|c|c|c|c|c|c|c|c|}
\hline & Gity & $\begin{array}{c}\text { Reve- } \\
\text { nues }\end{array}$ & $\begin{array}{l}\text { Expen- } \\
\text { ditures }\end{array}$ & $\begin{array}{l}\text { Surplus } \\
\text { /deficit }\end{array}$ & $\begin{array}{l}\text { Popula- } \\
\text { tion }\end{array}$ & $\begin{array}{l}\text { Reve- } \\
\text { nues }\end{array}$ & $\begin{array}{l}\text { Expen- } \\
\text { ditures }\end{array}$ & $\begin{array}{l}\text { Surplus } \\
\text { /deficit }\end{array}$ & $\begin{array}{l}\text { Trans- } \\
\text { paren- } \\
\text { cy }\end{array}$ \\
\hline 1 & Mali Lošinj & 11,211 & 8,951 & 2,260 & 8,116 & 91 & 73 & 18 & 5 \\
\hline 2 & Novalja & 11,041 & 11,493 & -452 & 3,663 & 40 & 42 & -2 & 2 \\
\hline 3 & Rovinj & 9,734 & 8,972 & 761 & 14,294 & 139 & 128 & 11 & 2 \\
\hline 4 & Opatija & 9,204 & 9,062 & 142 & 11,659 & 107 & 106 & 2 & 5 \\
\hline 5 & Krk & 9,085 & 9,624 & -539 & 6,281 & 57 & 60 & -3 & 4 \\
\hline 6 & Novigrad (Istra) & 8,976 & 9,171 & -194 & 4,345 & 39 & 40 & -1 & 4 \\
\hline 7 & Dubrovnik & 8,794 & 8,597 & 197 & 42,615 & 375 & 366 & 8 & 4 \\
\hline 8 & Poreč & 8,779 & 7,868 & 911 & 16,696 & 147 & 131 & 15 & 5 \\
\hline 9 & Cres & 8,129 & 9,530 & $-1,401$ & 2,879 & 23 & 27 & -4 & 5 \\
\hline 10 & Umag & 8,108 & 8,049 & 59 & 13,467 & 109 & 108 & 1 & 3 \\
\hline 11 & Zagreb & 7,856 & 7,719 & 137 & 790,017 & 6,206 & 6,098 & 108 & 5 \\
\hline 12 & Hvar & 7,823 & 8,555 & -732 & 4,251 & 33 & 36 & -3 & 4 \\
\hline 13 & Vis & 6,865 & 7,256 & -391 & 1,934 & 13 & 14 & -1 & 1 \\
\hline 14 & Crikvenica & 6,737 & 5,374 & 1,364 & 11,122 & 75 & 60 & 15 & 5 \\
\hline 15 & Bakar & 6,629 & 5,568 & 1,061 & 8,279 & 55 & 46 & 9 & 4 \\
\hline 16 & Vrlika & 6,627 & 6,324 & 304 & 2,177 & 14 & 14 & 1 & 1 \\
\hline 17 & Komiža & 6,604 & 6,550 & 55 & 1,526 & 10 & 10 & 0 & 3 \\
\hline 18 & Nin & 6,586 & 6,267 & 320 & 2,744 & 18 & 17 & 1 & 0 \\
\hline 19 & Vodnjan & 6,559 & 6,465 & 94 & 6,119 & 40 & 40 & 1 & 1 \\
\hline 20 & Biograd na Moru & 6,331 & 6,132 & 198 & 5,569 & 35 & 34 & 1 & 2 \\
\hline 21 & Pag & 6,045 & 6,279 & -234 & 3,846 & 23 & 24 & -1 & 1 \\
\hline 22 & Buzet & 5,880 & 6,686 & -805 & 6,133 & 36 & 41 & -5 & 5 \\
\hline 23 & Vodice & 5,850 & 5,414 & 436 & 8,875 & 52 & 48 & 4 & 5 \\
\hline 24 & Pazin & 5,701 & 5,443 & 257 & 8,638 & 49 & 47 & 2 & 5 \\
\hline 25 & Samobor & 5,597 & 5,487 & 110 & 37,633 & 211 & 207 & 4 & 3 \\
\hline 26 & Novi Vinodolski & 5,510 & 5,326 & 184 & 5,113 & 28 & 27 & 1 & 3 \\
\hline 27 & Supetar & 5,412 & 7,408 & $-1,996$ & 4,074 & 22 & 30 & -8 & 3 \\
\hline 28 & Labin & 5,363 & 5,236 & 127 & 11,642 & 62 & 61 & 1 & 5 \\
\hline 29 & Rijeka & 5,287 & 4,992 & 296 & 128,624 & 680 & 642 & 38 & 5 \\
\hline 30 & Stari Grad & 5,228 & 4,579 & 649 & 2,781 & 15 & 13 & 2 & 2 \\
\hline 31 & Obrovac & 5,062 & 4,957 & 105 & 4,323 & 22 & 21 & 0 & 1 \\
\hline 32 & Makarska & 4,865 & 4,251 & 615 & 13,834 & 67 & 59 & 9 & 4 \\
\hline 33 & Zaprešić & 4,819 & 4,538 & 281 & 25,223 & 122 & 114 & 7 & 4 \\
\hline 34 & Pula & 4,722 & 4,601 & 121 & 57,460 & 271 & 264 & 7 & 5 \\
\hline 35 & Zadar & 4,593 & 4,311 & 282 & 75,062 & 345 & 324 & 21 & 5 \\
\hline 36 & Buje & 4,579 & 4,932 & -353 & 5,182 & 24 & 26 & -2 & 4 \\
\hline 37 & Varaždin & 4,526 & 4,373 & 154 & 46,946 & 212 & 205 & 7 & 4 \\
\hline 38 & Gospić & 4,514 & 4,659 & -145 & 12,745 & 58 & 59 & -2 & 1 \\
\hline 39 & Senj & 4,461 & 4,073 & 388 & 7,182 & 32 & 29 & 3 & 3 \\
\hline 40 & Sveta Nedelja & 4,337 & 4,165 & 171 & 18,059 & 78 & 75 & 3 & 4 \\
\hline 41 & Koprivnica & 4,303 & 3,824 & 479 & 30,854 & 133 & 118 & 15 & 5 \\
\hline 42 & Rab & 4,303 & 3,615 & 688 & 8,065 & 35 & 29 & 6 & 4 \\
\hline 43 & Delnice & 4,207 & 5,028 & -821 & 5,952 & 25 & 30 & -5 & 4 \\
\hline 44 & Velika Gorica & 4,174 & 4,687 & -513 & 63,517 & 265 & 298 & -33 & 4 \\
\hline 45 & Vukovar & 4,006 & 4,493 & -487 & 27,683 & 111 & 124 & -13 & 4 \\
\hline 46 & Solin & 3,977 & 3,687 & 290 & 23,926 & 95 & 88 & 7 & 3 \\
\hline 47 & Virovitica & 3,895 & 4,147 & -252 & 21,291 & 83 & 88 & -5 & 4 \\
\hline 48 & Trogir & 3,882 & 3,392 & 490 & 13,192 & 51 & 45 & 6 & 4 \\
\hline 49 & Zabok & 3,801 & 4,024 & -223 & 8,994 & 34 & 36 & -2 & 3 \\
\hline 50 & Split & 3,799 & 3,759 & 39 & 178,102 & 677 & 670 & 7 & 5 \\
\hline 51 & Đurđevac & 3,797 & 3,758 & 39 & 8,264 & 31 & 31 & 0 & 3 \\
\hline 52 & Beli Manastir & 3,763 & 2,975 & 788 & 10,068 & 38 & 30 & 8 & 0 \\
\hline 53 & Čabar & 3,734 & 4,344 & -610 & 3,770 & 14 & 16 & -2 & 1 \\
\hline 54 & Čakovec & 3,651 & 3,777 & -126 & 27,104 & 99 & 102 & -3 & 4 \\
\hline
\end{tabular}

I2 NEWSLETTER Io8 | K. OTT, M. BRONIĆ, B. STANIĆ | Budget outturns of Croatian municipalities... | Institute of Public Finance 
Per capita (in HRK)

Total (in million HRK)

\begin{tabular}{|c|c|c|c|c|c|c|c|c|c|}
\hline & Gity & $\begin{array}{l}\text { Reve- } \\
\text { nues }\end{array}$ & $\begin{array}{l}\text { Expen- } \\
\text { ditures }\end{array}$ & $\begin{array}{l}\text { Surplus } \\
\text { /deficit }\end{array}$ & $\begin{array}{l}\text { Popula- } \\
\text { tion }\end{array}$ & $\begin{array}{l}\text { Reve- } \\
\text { nues }\end{array}$ & $\begin{array}{l}\text { Expen- } \\
\text { ditures }\end{array}$ & $\begin{array}{l}\text { Surplus } \\
\text { /deficit }\end{array}$ & $\begin{array}{l}\text { Trans- } \\
\text { paren- } \\
\text { cy }\end{array}$ \\
\hline 55 & Kastav & 3,605 & 3,031 & 574 & 10,440 & 38 & 32 & 6 & 5 \\
\hline 56 & $\begin{array}{l}\text { Hrvatska } \\
\text { Kostajnica }\end{array}$ & 3,590 & 3,712 & -122 & 2,756 & 10 & 10 & 0 & 4 \\
\hline 57 & Šibenik & 3,527 & 3,814 & -287 & 46,332 & 163 & 177 & -13 & 5 \\
\hline 58 & Kraljevica & 3,526 & 3,324 & 203 & 4,618 & 16 & 15 & 1 & 2 \\
\hline 59 & Karlovac & 3,516 & 3,626 & -110 & 55,705 & 196 & 202 & -6 & 5 \\
\hline 60 & Lipik & 3,498 & 3,521 & -24 & 6,170 & 22 & 22 & 0 & 4 \\
\hline 61 & Sisak & 3,492 & 3,435 & 57 & 47,768 & 167 & 164 & 3 & 4 \\
\hline 62 & Korčula & 3,443 & 3,516 & -73 & 5,663 & 19 & 20 & 0 & 4 \\
\hline 63 & Drniš & 3,439 & 3,551 & -112 & 7,498 & 26 & 27 & -1 & 4 \\
\hline 64 & Ivanić-Grad & 3,431 & 3,006 & 424 & 14,548 & 50 & 44 & 6 & 4 \\
\hline 65 & Nova Gradiška & 3,223 & 3,119 & 104 & 14,229 & 46 & 44 & 1 & 2 \\
\hline 66 & Osijek & 3,200 & 3,172 & 28 & 108,048 & 346 & 343 & 3 & 5 \\
\hline 67 & Omiš & 3,187 & 3,063 & 124 & 14,936 & 48 & 46 & 2 & 3 \\
\hline 68 & Kutina & 3,180 & 2,975 & 205 & 22,760 & 72 & 68 & 5 & 3 \\
\hline 69 & Belišće & 3,084 & 3,474 & -390 & 10,825 & 33 & 38 & -4 & 1 \\
\hline 70 & Novska & 3,072 & 3,102 & -30 & 13,518 & 42 & 42 & 0 & 3 \\
\hline 71 & Skradin & 3,018 & 3,002 & 16 & 3,825 & 12 & 11 & 0 & 1 \\
\hline 72 & Vrbovsko & 3,009 & 3,053 & -44 & 5,076 & 15 & 15 & 0 & 3 \\
\hline 73 & Jastrebarsko & 2,994 & 3,006 & -12 & 15,866 & 48 & 48 & 0 & 4 \\
\hline 74 & Petrinja & 2,795 & 3,057 & -262 & 24,671 & 69 & 75 & -6 & 3 \\
\hline 75 & Orahovica & 2,793 & 2,863 & -70 & 5,304 & 15 & 15 & 0 & 4 \\
\hline 76 & Prelog & 2,765 & 2,992 & -228 & 7,815 & 22 & 23 & -2 & 3 \\
\hline 77 & Trilj & 2,753 & 2,770 & -16 & 9,109 & 25 & 25 & 0 & 1 \\
\hline 78 & Slunj & 2,749 & 2,730 & 20 & 5,076 & 14 & 14 & 0 & 4 \\
\hline 79 & Kaštela & 2,724 & 2,716 & 8 & 38,667 & 105 & 105 & 0 & 4 \\
\hline 80 & Opuzen & 2,714 & 2,454 & 260 & 3,254 & 9 & 8 & 1 & 3 \\
\hline 81 & Grubišno Polje & 2,710 & 3,175 & -465 & 6,478 & 18 & 21 & -3 & 2 \\
\hline 82 & Ludbreg & 2,678 & 3,069 & -391 & 8,478 & 23 & 26 & -3 & 2 \\
\hline 83 & Ogulin & 2,674 & 2,786 & -112 & 13,915 & 37 & 39 & -2 & 4 \\
\hline 84 & Bjelovar & 2,659 & 2,719 & -60 & 40,276 & 107 & 110 & -2 & 5 \\
\hline 85 & Ploče & 2,659 & 2,201 & 458 & 10,135 & 27 & 22 & 5 & 2 \\
\hline 86 & Otočac & 2,650 & 2,303 & 347 & 9,778 & 26 & 23 & 3 & 2 \\
\hline 87 & Križevci & 2,632 & 2,501 & 132 & 21,122 & 56 & 53 & 3 & 4 \\
\hline 88 & Popovača & 2,626 & 2,707 & -81 & 11,905 & 31 & 32 & -1 & 1 \\
\hline 89 & Krapina & 2,582 & 2,412 & 170 & 12,480 & 32 & 30 & 2 & 3 \\
\hline 90 & Našice & 2,512 & 2,894 & -382 & 16,224 & 41 & 47 & -6 & 1 \\
\hline 91 & Čazma & 2,493 & 2,070 & 422 & 8,077 & 20 & 17 & 3 & 1 \\
\hline 92 & Požega & 2,472 & 2,320 & 152 & 26,248 & 65 & 61 & 4 & 3 \\
\hline 93 & Imotski & 2,429 & 2,459 & -29 & 10,764 & 26 & 26 & 0 & 0 \\
\hline 94 & Vinkovci & 2,428 & 2,284 & 144 & 35,312 & 86 & 81 & 5 & 0 \\
\hline 95 & Glina & 2,424 & 2,633 & -209 & 9,283 & 23 & 24 & -2 & 4 \\
\hline 96 & Županja & 2,392 & 2,399 & -7 & 12,090 & 29 & 29 & 0 & 5 \\
\hline 97 & Slatina & 2,384 & 2,136 & 247 & 13,686 & 33 & 29 & 3 & 3 \\
\hline 98 & Pakrac & 2,343 & 2,199 & 144 & 8,460 & 20 & 19 & 1 & 3 \\
\hline 99 & Slavonski Brod & 2,314 & 2,055 & 259 & 59,141 & 137 & 122 & 15 & 5 \\
\hline 100 & Knin & 2,292 & 2,550 & -258 & 15,407 & 35 & 39 & -4 & 1 \\
\hline 101 & Vrbovec & 2,287 & 2,087 & 201 & 14,797 & 34 & 31 & 3 & 3 \\
\hline 102 & Otok (Vinkovci) & 2,228 & 2,408 & -180 & 6,343 & 14 & 15 & -1 & 1 \\
\hline 103 & Benkovac & 2,222 & 2,154 & 69 & 11,026 & 25 & 24 & 1 & 2 \\
\hline 104 & Dugo Selo & 2,213 & 2,225 & -11 & 17,466 & 39 & 39 & 0 & 3 \\
\hline 105 & Daruvar & 2,208 & 2,133 & 75 & 11,633 & 26 & 25 & 1 & 3 \\
\hline 106 & Garešnica & 2,208 & 2,093 & 115 & 10,472 & 23 & 22 & 1 & 4 \\
\hline 107 & Mursko Središće & 2,185 & 1,916 & 269 & 6,307 & 14 & 12 & 2 & 2 \\
\hline 108 & Ilok & 2,146 & 2,050 & 96 & 6,767 & 15 & 14 & 1 & 0 \\
\hline 109 & Oroslavje & 2,111 & 2,064 & 47 & 6,138 & 13 & 13 & 0 & 1 \\
\hline 110 & Klanjec & 2,052 & 2,463 & -412 & 2,915 & 6 & 7 & -1 & 4 \\
\hline
\end{tabular}

NEWSLETTER IO8 | K. OTT, M. BRONIĆ, B. STANIĆ | Budget outturns of Croatian municipalities... | Institute of Public Finance I3 
Per capita (in HRK)

Total (in million HRK)

\begin{tabular}{|c|c|c|c|c|c|c|c|c|c|}
\hline & Gity & $\begin{array}{l}\text { Reve- } \\
\text { nues }\end{array}$ & $\begin{array}{l}\text { Expen- } \\
\text { ditures }\end{array}$ & $\begin{array}{l}\text { Surplus } \\
\text { /deficit }\end{array}$ & $\begin{array}{l}\text { Popula- } \\
\text { tion }\end{array}$ & $\begin{array}{l}\text { Reve- } \\
\text { nues }\end{array}$ & $\begin{array}{l}\text { Expen- } \\
\text { ditures }\end{array}$ & $\begin{array}{l}\text { Surplus } \\
\text { /deficit }\end{array}$ & $\begin{array}{l}\text { Trans- } \\
\text { paren- } \\
\text { cy }\end{array}$ \\
\hline 111 & Vrgorac & 1,986 & 2,193 & -207 & 6,572 & 13 & 14 & -1 & 0 \\
\hline 112 & SvetiIvan Zelina & 1,964 & 1,761 & 203 & 15,959 & 31 & 28 & 3 & 0 \\
\hline 113 & Sinj & 1,945 & 1,984 & -39 & 24,826 & 48 & 49 & -1 & 1 \\
\hline 114 & Lepoglava & 1,915 & 2,385 & -470 & 8,283 & 16 & 20 & -4 & 4 \\
\hline 115 & Zlatar & 1,899 & 1,213 & 686 & 6,096 & 12 & 7 & 4 & 4 \\
\hline 116 & Đakovo & 1,889 & 1,459 & 429 & 27,745 & 52 & 40 & 12 & 1 \\
\hline 117 & Ozalj & 1,879 & 2,500 & -622 & 6,817 & 13 & 17 & -4 & 5 \\
\hline 118 & Donja Stubica & 1,848 & 1,642 & 205 & 5,680 & 10 & 9 & 1 & 3 \\
\hline 119 & Duga Resa & 1,809 & 1,822 & -13 & 11,180 & 20 & 20 & 0 & 5 \\
\hline 120 & Pleternica & 1,759 & 1,805 & -45 & 11,323 & 20 & 20 & -1 & 0 \\
\hline 121 & Ivanec & 1,745 & 1,856 & -111 & 13,758 & 24 & 26 & -2 & 5 \\
\hline 122 & Metković & 1,729 & 1,634 & 95 & 16,788 & 29 & 27 & 2 & 3 \\
\hline 123 & Pregrada & 1,680 & 1,535 & 145 & 6,594 & 11 & 10 & 1 & 4 \\
\hline 124 & Valpovo & 1,671 & 1,650 & 22 & 11,563 & 19 & 19 & 0 & 1 \\
\hline 125 & $\begin{array}{l}\text { Varaždinske } \\
\text { Toplice }\end{array}$ & 1,669 & 1,831 & -162 & 6,364 & 11 & 12 & -1 & 3 \\
\hline 126 & Donji Miholjac & 1,484 & 1,504 & -19 & 9,491 & 14 & 14 & 0 & 3 \\
\hline 127 & Kutjevo & 1,415 & 1,667 & -251 & 6,247 & 9 & 10 & -2 & 0 \\
\hline 128 & Novi Marof & 1,292 & 1,173 & 120 & 13,246 & 17 & 16 & 2 & 2 \\
\hline
\end{tabular}

Source: Authors' data based on: Ministry of Finance (20I6), CBS (2016) and Ott et al. (2016).

Table 3.

Budget outturns of counties, 2015.

Per capita (in HRK)

Total (in million HRK)

\begin{tabular}{|c|c|c|c|c|c|c|c|c|c|}
\hline & County & $\begin{array}{l}\text { Reve- } \\
\text { nues }\end{array}$ & $\begin{array}{l}\text { Expen- } \\
\text { ditures }\end{array}$ & $\begin{array}{l}\text { Surplus } \\
\text { /deficit }\end{array}$ & $\begin{array}{l}\text { Popula- } \\
\text { tion }\end{array}$ & $\begin{array}{c}\text { Reve- } \\
\text { nues }\end{array}$ & $\begin{array}{l}\text { Expen- } \\
\text { ditures }\end{array}$ & $\begin{array}{l}\text { Surplus } \\
\text { /deficit }\end{array}$ & $\begin{array}{c}\text { Trans- } \\
\text { paren- } \\
\text { cy }\end{array}$ \\
\hline 1 & Lika-Senj & 1,613 & 1,172 & 441 & 50,927 & 82 & 60 & 22 & 5 \\
\hline 2 & $\begin{array}{l}\text { Virovitica- } \\
\text { Podravina }\end{array}$ & 1,446 & 1,468 & -22 & 84,836 & 123 & 125 & -2 & 4 \\
\hline 3 & $\begin{array}{l}\text { Dubrovnik- } \\
\text { Neretva }\end{array}$ & 1,256 & 1,265 & -8 & 122,568 & 154 & 155 & -1 & 5 \\
\hline 4 & Istria & 1,242 & 1,238 & 4 & 208,055 & 258 & 258 & 1 & 5 \\
\hline 5 & Osijek-Baranja & 1,222 & 971 & 251 & 305,032 & 373 & 296 & 77 & 4 \\
\hline 6 & Sisak-Moslavina & 1,175 & 1,255 & -80 & 172,439 & 203 & 216 & -14 & 2 \\
\hline 7 & Varaždin & 1,150 & 1,135 & 16 & 175,951 & 202 & 200 & 3 & 5 \\
\hline 8 & $\begin{array}{l}\text { Koprivnica- } \\
\text { Križevci }\end{array}$ & 1,108 & 1,160 & -53 & 115,584 & 128 & 134 & -6 & 4 \\
\hline 9 & Karlovac & 1,102 & 1,098 & 4 & 128,899 & 142 & 142 & 1 & 5 \\
\hline 10 & Međimurje & 1,101 & 1,105 & -3 & 113,804 & 125 & 126 & 0 & 3 \\
\hline 11 & Krapina-Zagorje & 1,093 & 1,099 & -6 & 132,892 & 145 & 146 & -1 & 5 \\
\hline 12 & Požega-Slavonia & 1,090 & 1,005 & 85 & 78,034 & 85 & 78 & 7 & 2 \\
\hline 13 & $\begin{array}{l}\text { Primorje-Gorski } \\
\text { Kotar }\end{array}$ & 1,077 & 1,107 & -31 & 296,195 & 319 & 328 & -9 & 4 \\
\hline 14 & Zadar & 1,016 & 1,089 & -73 & 170,017 & 173 & 185 & -12 & 5 \\
\hline 15 & Šibenik-Knin & 1,006 & 1,011 & -5 & 109,375 & 110 & 111 & -1 & 5 \\
\hline 16 & Bjelovar-Bilogora & 940 & 920 & 20 & 119,764 & 113 & 110 & 2 & 5 \\
\hline 17 & Vukovar-Srijem & 923 & 896 & 27 & 179,521 & 166 & 161 & 5 & 4 \\
\hline 18 & Split-Dalmatia & 887 & 873 & 15 & 454,798 & 404 & 397 & 7 & 4 \\
\hline 19 & Zagreb & 868 & 838 & 30 & 317,606 & 276 & 266 & 10 & 5 \\
\hline 20 & Brod-Posavina & 859 & 845 & 14 & 158,575 & 136 & 134 & 2 & 5 \\
\hline
\end{tabular}

Source: Authors' data based on: Ministry of Finance (20I6), CBS (2016) and Ott et al. (2016).

I4 NEWSLETTER IO8 | K. OTT, M. BRONIĆ, B. STANIĆ | Budget outturns of Croatian municipalities... | Institute of Public Finance 